\title{
SINGULAR POINTS OF THE SUM OF A SERIES OF EXPONENTIAL MONOMIALS ON THE BOUNDARY OF THE CONVERGENCE DOMAIN
}

\author{
O. A. KRIVOSHEYEVA
}

\begin{abstract}
Singular points for the sum of a series of exponential monomials are studied. The main statement contains results of Hadamard, Fabry, V. Bernstein, Polya, Carlson and Landau as particular cases. Moreover, a special function is constructed that has no singular points on the boundary of the convergence domain of its series. This function generalizes a certain special function in the theory of Dirichlet series to the case of series of exponential monomials. The existence of this special function shows the necessity of a condition in the main theorem; in V. Bernstein's theorem, a similar role is played by the requirement that the condensation index should be equal to zero.
\end{abstract}

\section{$\S 1$. INTRODUCTION}

Let $\Lambda=\left\{\lambda_{k}, m_{k}\right\}_{k=1}^{\infty}$ be a multiple sequence, where the $\lambda_{k}$ are complex numbers whose moduli are monotone nondecreasing with $k,\left|\lambda_{k}\right| \rightarrow \infty$, and the $m_{k}$ are natural numbers. We consider the series of exponential monomials generated by $\Lambda$, i.e., the series of the form

$$
\sum_{k=1, n=0}^{\infty, m_{k}-1} d_{k, n} z^{n} \exp \left(\lambda_{k} z\right)
$$

The distribution of singular points for the sum of this series on the boundary of the domain of convergence is studied.

Let $d=\left\{d_{k, n}\right\}_{k=1, n=0}^{\infty, m_{k}-1}$ be a sequence of complex numbers. We denote by $g_{d}(z)$ the sum of the series (1.1), and by $D(\Lambda, d)$ the interior of the set of all points $z \in \mathbb{C}$ at which the series converges. Generally speaking, the set $D(\Lambda, d)$ may fail to be convex (see [1]) or even connected (see [2]). But if

$$
m(\Lambda)=\limsup _{k \rightarrow \infty} \frac{m_{k}}{\left|\lambda_{k}\right|}=0, \quad \sigma(\Lambda)=\limsup _{j \rightarrow \infty} \frac{\ln j}{\xi_{j}}=0,
$$

where $\left\{\xi_{j}\right\}$ is the sequence with monotone nondecreasing moduli formed by the $\lambda_{k}$ in such a way that each $\lambda_{k}$ occurs precisely $m_{k}$ times, then the Cauchy-Hadamard theorem for series of exponential monomials (see [2]) shows that $D(\Lambda, d)$ is a convex domain (which may be empty) admitting a description in terms of the coefficients $\left\{d_{k, n}\right\}$. Moreover, under the same conditions, by the Abel theorem (see [2]) for series of this sort, the expansion (1.1) converges absolutely and uniformly on every compact subset of $D(\Lambda, d)$. In particular, its sum $g_{d}(z)$ is analytic in $D(\Lambda, d)$.

We shall denote by $\mathfrak{A}(\Lambda)$ the set of all coefficient sequences $d=\left\{d_{k, n}\right\}_{k=1, n=0}^{\infty, m_{k}-1}$ for the series (1.1) such that the set $D(\Lambda, d)$ is not empty and the functions $g_{d}(z)$ are analytic

2010 Mathematics Subject Classification. Primary 30B50.

Key words and phrases. Series of exponentials, convex domain, singular point. 
in $D(\Lambda, d)$. Let $d \in \mathfrak{A}(\Lambda)$. A point $z \in \partial D(\Lambda, d)$ is said to be singular for $g_{d}(z)$ if this function does not admit analytic continuation to any domain containing $z$ and including $D(\Lambda, d)$.

The problem of describing the singular points on the boundary of $D(\Lambda, d)$ counts a long history. It originates in the investigation (started as early as in the 19th century) of domains of existence for functions representable by power series. They are a partial case of the Dirichlet series, specifically, of the series (1.1) with $m_{k} \equiv 1$ and $\lambda_{k}$ negative. The substitution $w=\exp (-z)$ converts a power series into a Dirichlet series. In 1892, Hadamard proved that if $g$ is representable by a power series

$$
g(w)=\sum_{n=1}^{\infty} d_{n} w^{k(n)}
$$

with gaps, $k(n+1)-k(n) \geq \alpha k(n)$, where $\alpha$ is a positive number independent of $n$, then the boundary of the convergence disk for this series is the natural boundary of the domain of existence for $g$, i.e., every point of this boundary is singular for $g$. In 1896, Fabry 4 proved (among other things) that Hadamard's theorem remains true under a less restrictive condition on the sequence of exponents, namely, under the requirement that the sequence $\{k(n)\}$ have zero density:

$$
\lim _{n \rightarrow \infty} \frac{n}{k(n)}=0
$$

Polya [5, 6] and also Carlson and Landau (see, e.g., 7, Chapter II, §5.2]) extended this result to the case of Dirichlet series. They showed that if $g$ is representable by a Dirichlet series

$$
g(z)=\sum_{k=1}^{\infty} d_{k} \exp \left(-\lambda_{k} z\right)
$$

with a sequence $\Lambda=\left\{\lambda_{k}\right\}$ of positive exponents that has zero density and satisfies $\lambda_{k+1}-\lambda_{k} \geq h>0, k=1,2, \ldots$, then either $g(z)$ is an entire function or the convergence line (the vertical line that bounds the half-plane of convergence for the Dirichlet series) is the natural boundary of the domain of existence for $g$. This result is a partial case of a more general statement by $\mathrm{V}$. Bernstein, see [8]. He proved that, under the conditions

where

$$
\lim _{k \rightarrow \infty} \frac{k}{\lambda_{k}}=\tau, \quad \gamma(\Lambda)=\limsup _{k \rightarrow \infty} \frac{1}{\lambda_{k}} \ln \left|\frac{1}{L^{\prime}\left(\lambda_{k}\right)}\right|=0,
$$

$$
L(\lambda)=\prod_{k=1}^{\infty}\left(1-\frac{\lambda^{2}}{\lambda_{k}^{2}}\right),
$$

every interval of length $2 \pi$ on the convergence line for the series (1.3) (if this line exists) contains at least one singular point for $g(z)$. In particular, this implies a refinement of the Fabry theorem: if the sequence $\{k(n)\}$ for the series (1.2) has density $\tau=\lim n / k(n)$, then every closed arc of length $2 \pi \tau$ on the circle of convergence contains at least one singular point for $g(w)$. It should be noted that Polya had proved V. Bernstein's statement prior to him, but under a stronger restriction (compared to $\gamma(\Lambda)=0$ ) on the sequence $\Lambda=\left\{\lambda_{k}\right\}: \lambda_{k+1}-\lambda_{k} \geq h>0, k=1,2, \ldots$ In [9, Chapter II, §3.3] a special function was constructed which is the sum of a Dirichlet series and, together with $\gamma(\Lambda) \neq 0$, has no singular points on the convergence line. The existence of such a function shows that the condition $\gamma(\Lambda)=0$ is necessary in V. Bernstein's theorem.

Leont'ev 9] generalized the results of Fabry, Polya, and V. Bernstein to the case of series of exponentials (i.e., series of the form (1.1) with $m_{k} \equiv 1$ ) whose sequence of exponents has zero density. He proved that, under this condition and the supplementary 
condition $\gamma(\Lambda)=0$, the domain of convergence for the series of exponentials coincides with the domain of analyticity for the sum of this series. In particular, this implies that the latter domain is convex.

In connection with the problem under discussion, we mention also the papers [10 by Ostrowski and 11, 12] by Lunts, which stay somewhat apart. In the first paper, Dirichlet series were studied whose sequence of exponents has finite upper density:

$$
\limsup _{k \rightarrow \infty} \frac{k}{\lambda_{k}}=\alpha>0 \text {. }
$$

A well-known theorem by Ostrowski (see also [20] and [7, Theorem 2.4.7]) says that, under the condition

$$
\liminf _{k \rightarrow \infty}\left(\lambda_{k+1}-\lambda_{k}\right)=h,
$$

the function (1.3) has a singular point in every closed disk of radius $r(\alpha, h)=\pi \alpha^{*}+$ $3(3-\ln (h \alpha)) \alpha$ and centered at the convergence line. Here $\alpha^{*}$ is the averaged upper density of the sequence $\left\{\lambda_{k}\right\}$ (see [7); it satisfies the estimates $\alpha^{*} \leq \alpha \leq e \alpha^{*}$. Note that the definition of the upper density implies the inequality $\alpha \leq 1 / h$. Under condition (1.4), the sequence $\left\{\lambda_{k}\right\}$ can be completed up to a sequence with density not exceeding 1/h (see, e.g., [1]). By Polya's (or Bernstein's) theorem, the function (1.3) has at least one singular point on each interval of lengths at least $2 \pi / h$ on the convergence line. If the upper density $\alpha$ is not too distant from $1 / h$, then $2 \pi / h$ is much smaller than $r(\alpha, h)$. In this case the Polya theorem yields more than that of Ostrowski. So, in the context of the problem under study, the results of [10] become substantial only for Dirichlet series of special type, when the upper density $\alpha$ of the sequence $\left\{\lambda_{k}\right\}$ is much smaller than $1 / h$. In this case, the exponents of the series must mainly be concentrated in groups that lie fairly far from one another on the line. In this connection, a natural question arises about the applicability of Ostrowski's result to the problem of the distribution of singular points for the sum of a Dirichlet series on its convergence line.

In the papers by Lunts, more general series of exponentials were treated. He proved interesting and subtle results about the distribution of singular points on the boundary of the convergence domain for a series of exponentials. However, the singular points of all "partial sums" $g(z, \Gamma)$ of this series were treated rather than those of its sum $g(z)$. The function $g(z, \Gamma)$ is the sum of the terms of the series whose exponents lie in the angle $\Gamma$. The set of all singular points of the functions $g(z, \Gamma)$ (including $g(z)=g(z, \mathbb{C})$ ) is much larger than that of $g(z)$. As an example, consider the series

$$
\sum_{k=1}^{\infty} \frac{\exp \left(\lambda_{k} z\right)}{L^{\prime}\left(\lambda_{k}\right)}
$$

where

$$
L(\lambda)=\frac{\sin \lambda \sin (i \lambda)}{\lambda^{2}}
$$

and $\Lambda=\left\{\lambda_{k}\right\}$ is the sequence of zeros of $L(\lambda)$ formed by the points $\pm \pi n$ and $\pm i \pi n$, $n=1,2, \ldots$, on the real and the imaginary axis. The domain of convergence of the series (1.5) is the square with vertices at $1+i, i-1,1-i,-1-i$, but its sum is identically zero, consequently, has no singular points (see [9. Chapter II, §2.3]). At the same time, each of the four "partial sums" $g(z, \Gamma)$ for the series (1.5) that correspond to the $\lambda_{k}$ lying on a particular coordinate semiaxis, has at least one singular point on the side of the square that corresponds to this semiaxis. Indeed, let $g(z, \Gamma)$ be the sum of the Dirichlet series corresponding to the negative $\lambda_{k}$. The sequence of exponents for this series has density $\tau=1 / \pi$ and the distance between neighboring exponents is 1 . Then, by Polya's theorem cited above, the function $g(z, \Gamma)$ has at least one singular point on each interval 
of length 2 on the convergence line (the vertical line containing the interval $[-1-i, i-1]$ ). The same is applicable to the remaining three partial sums of the series (1.5).

In this paper, singular points for a general series of the form (1.1) are studied. The result obtained implies the statements mentioned above except for those in the last three papers. Moreover, a special function is constructed that has no singularities on the boundary of the convergence domain for its series. This function is a generalization of the special function in the theory of Dirichlet series mentioned above to the case of series of exponential monomials. Its existence proves the necessity of an assumption in the main theorem (this assumption is similar in nature to the condition $\gamma(\Lambda)=0$ in Bernstein's theorem). In the next to the last section of the paper, a complete answer is given to the question about conditions that guarantee that the domain of existence for the sum of the series (1.1) is convex and coincides with the domain where the series converges. As a consequence, the converse to Fabry's theorem mentioned above is proved. It is spectacular that the condition $\lim _{n \rightarrow \infty} n / k(n)=0$ is not only sufficient but also necessary for the domain of the sum of an arbitrary series (1.2) to coincide with the convergence disk for this series. This statement is a particular case of more general results by Fuchs and Malliavin (see [21]). Finally, in the last section, the question mentioned above about Ostrowski's theorem is answered. By an example, we show that, under the assumptions of that theorem, the distance between singular points of the sum of a Dirichlet series on its convergence line may even be like $O(1 / h)$ (rather than like $O(-\ln h$ ), as we have for $r(\alpha, h))$. This means that, in essence, the Ostrowski theorem is related to singular points of the sum of a Dirichlet series that lie in a neighborhood of the convergence line rather than on this line itself. Moreover, the example in question provides a lower estimate for the size of this neighborhood. It turns out that the upper density $\alpha$ of the sequence $\left\{\lambda_{k}\right\}$ is responsible for this estimate.

\section{§2. Characteristics of a Complex sequence}

In this section, we consider some well-known characteristics for the distribution of points of a complex sequence, and study their interrelations.

As in [13, 14, for a sequence $\Lambda=\left\{\lambda_{k}, m_{k}\right\}_{k=1}^{\infty}$, we introduce a quantity describing how densely the points $\lambda_{k}$ are situated. We put

$$
\begin{aligned}
q_{\Lambda}(z, w, \delta) & =\prod_{\lambda_{k} \in B(w, \delta|w|)}\left(\frac{z-\lambda_{k}}{3 \delta\left|\lambda_{k}\right|}\right)^{m_{k}}, \\
q_{\Lambda}^{j}(z, \delta) & =q_{\Lambda}\left(z, \lambda_{j}, \delta\right)\left(\frac{z-\lambda_{j}}{3 \delta\left|\lambda_{j}\right|}\right)^{-m_{j}} .
\end{aligned}
$$

Here $B(w, r)$ is the open disk centered at $w$ and of radius $r$. The modulus of the function $q_{\Lambda}(z, w, \delta)$ can be interpreted as the measure of concentration of the points $\lambda_{k} \in B(w, \delta|w|)$ near $z$. If the disk $B(w, \delta|w|)$ does not contain any $\lambda_{k}$, we agree that $q_{\Lambda}(z, w, \delta) \equiv 1$. It should be noted that, on the disk $B(w, \delta|w|)$, the absolute value of each factor in the definition of $q_{\Lambda}$ does not exceed the quantity $2(3(1-\delta))^{-1}$ (for $\delta \in(0,1))$, i.e., it is less than or equal to 1 if $\delta \in(0,1 / 3)$. Moreover, if $\delta_{1} \leq \delta_{2}$ and $B\left(w_{1}, \delta_{1}\left|w_{1}\right|\right) \subseteq B\left(w_{2}, \delta_{2}\left|w_{2}\right|\right)$, then the number of factors in the definition of $q_{\Lambda}\left(z, w_{1}, \delta_{1}\right)$ does not exceed that in the definition of $q_{\Lambda}\left(z, w_{2}, \delta_{2}\right)$. Thus,

$$
\left|q_{\Lambda}\left(z, w_{1}, \delta_{1}\right)\right| \geq\left|q_{\Lambda}\left(z, w_{2}, \delta_{2}\right)\right|, \quad z \in \mathbb{C},
$$

if $0<\delta_{1} \leq \delta_{2}<1 / 3$ and $B\left(w_{1}, \delta_{1}\left|w_{1}\right|\right) \subseteq B\left(w_{2}, \delta_{2}\left|w_{2}\right|\right)$. A similar inequality is true for $q_{\Lambda}^{j}(z, \delta)$ if $0<\delta_{1} \leq \delta_{2}<1 / 3$ :

$$
\left|q_{\Lambda}^{j}\left(z, \delta_{1}\right)\right| \geq\left|q_{\Lambda}^{j}\left(z, \delta_{2}\right)\right|, \quad z \in \mathbb{C} .
$$


We put $S_{\Lambda}=0$ if $\Lambda$ is finite and

$$
S_{\Lambda}=\lim _{\delta \rightarrow 0} \liminf _{k \rightarrow \infty} \frac{\ln \left|q_{\Lambda}^{k}\left(\lambda_{k}, \delta\right)\right|}{\left|\lambda_{k}\right|}
$$

otherwise. This definition is consistent because, by (2.1), the limit as $\delta \rightarrow 0$ always exists. By the said above, we have $S_{\Lambda} \leq 0$. The quantity $S_{\Lambda}$ resembles somewhat the classical condensation index of $\Lambda$, see [7, 15]. As in [14], we introduce also the quantity

$$
M_{\Lambda}=\lim _{\delta \rightarrow 0} \limsup _{k \rightarrow \infty} \frac{M_{\Lambda}\left(\lambda_{k}, \delta\right)}{\left|\lambda_{k}\right|}
$$

where $M_{\Lambda}(w, \delta)=\sum_{\lambda_{k} \in B(w, \delta|w|)} m_{k}$. We agree that $M_{\Lambda}=0$ if $\Lambda$ is finite. Clearly, $M_{\Lambda} \geq 0$ and

$$
M_{\Lambda}\left(w_{1}, \delta_{1}\right) \leq M_{\Lambda}\left(w_{2}, \delta_{2}\right) \text { if } B\left(w_{1}, \delta_{1}\left|w_{1}\right|\right) \subseteq B\left(w_{2}, \delta_{2}\left|w_{2}\right|\right) .
$$

This implies that $M_{\Lambda}$ is well defined. It can easily be observed that $m(\Lambda) \leq M_{\Lambda}$ (the quantity $m(\Lambda)$ was defined in the Introduction).

Suppose that the sequence $\Lambda$ has finite upper density, i.e.,

$$
N(\Lambda)=\limsup _{l \rightarrow \infty} \frac{l}{\left|\eta_{l}\right|}<\infty
$$

where the sequence $\left\{\eta_{l}\right\}$ is built of the points $\left\{\lambda_{k}\right\}$ and each $\lambda_{k}$ occurs precisely $m_{k}$ times in this sequence. This assumption is equivalent (see, e.g., [15]) to the statement that $\Lambda$ constitutes part of the zeros of an entire function $f$ of exponential type. In other words, $f$ vanishes at each $\lambda_{k}$ with multiplicity of at least $m_{k}$. It is easily seen that $M_{\Lambda} \leq N(\Lambda)$.

We illustrate the quantities introduced above by an example. Put $\lambda_{2 k}=k$ and $\lambda_{2 k-1}=k-e^{-\varepsilon k}, k=1,2, \ldots$, where $\varepsilon>0$. It is easily seen that, in this case, $N(\Lambda)=2$ and

$$
M_{\Lambda} \leq \lim _{\delta \rightarrow 0} \limsup _{k \rightarrow \infty} \frac{2 \delta\left|\lambda_{k}\right|}{\left|\lambda_{k}\right|}=0 .
$$

We calculate $S_{\Lambda}$. Let $\delta \in(0,1 / 3)$. Then the numbers $\left(z-\lambda_{k}\right) / 3 \delta\left|\lambda_{k}\right|$ do not exceed 1 in modulus, so we have

$$
\left|q_{\Lambda}^{2 k}\left(\lambda_{2 k}, \delta\right)\right| \leq\left|\left(\frac{\lambda_{2 k}-\lambda_{2 k-1}}{3 \delta\left|\lambda_{2 k-1}\right|}\right)\right| \leq \frac{e^{-\varepsilon k}}{3 \delta\left(k-e^{-\varepsilon k}\right)} .
$$

Consequently,

$$
S_{\Lambda}=\lim _{\delta \rightarrow 0} \liminf _{l \rightarrow \infty} \frac{\ln \left|q_{\Lambda}^{l}\left(\lambda_{l}, \delta\right)\right|}{\left|\lambda_{l}\right|} \leq \lim _{\delta \rightarrow 0} \liminf _{k \rightarrow \infty} k^{-1} \ln \left(\frac{e^{-\varepsilon k}}{3 \delta\left(k-e^{-\varepsilon k}\right)}\right)=-\varepsilon .
$$

Thus, in this example the quantity $S_{\Lambda}$ is negative and characterizes the distance between the neighboring points $\lambda_{2 k}$ and $\lambda_{2 k-1}$. This distance tends to zero like $e^{-\varepsilon k}$, where $-\varepsilon \geq S_{\Lambda}$. The next example shows that the mere convergence to zero for the distance between neighboring points does not guarantee that $S_{\Lambda}$ is negative.

This time we take $\lambda_{2 k}=k$ and $\lambda_{2 k-1}=k-e^{-\varepsilon(k) k}, k=1,2, \ldots$, where $\varepsilon(k) \rightarrow 0$ and $e^{-\varepsilon(k) k} \rightarrow 0$ as $k \rightarrow \infty$ (for instance, we may put $\varepsilon(k)=1 / \sqrt{k}$ ). The quantities $N(\Lambda)$ and $M_{\Lambda}$ are the same as in the preceding example. We find $S_{\Lambda}$. We have

$$
\ln \left|q_{\Lambda}^{2 k}\left(\lambda_{2 k}, \delta\right)\right| \geq \sum_{\substack{\lambda_{l} \in B\left(\lambda_{2 k}, \delta\left|\lambda_{2 k}\right|\right) \\ \lambda_{l} \neq \lambda_{2 k}}} \ln \left|\frac{\lambda_{2 k}-\lambda_{l}}{3 \delta\left|\lambda_{l}\right|}\right| .
$$

Let $\delta \in(0,1 / 3)$. Then all summands on the right in this inequality are negative. Increasing their number will not destroy the inequality. So, if we add (if necessary) the 
summands constructed by the $\lambda_{l}$ that lie in $B\left(\lambda_{2 k}, \delta\left|\lambda_{2 k}\right|+2\right)$ but outside $B\left(\lambda_{2 k}, \delta\left|\lambda_{2 k}\right|\right)$, we obtain

$$
\ln \left|q_{\Lambda}^{2 k}\left(\lambda_{2 k}, \delta\right)\right| \geq \sum_{-2 r(k)-1 \leq l \leq 2 r(k)} \ln \left|\frac{\lambda_{2 k}-\lambda_{2 k+l}}{3 \delta\left|\lambda_{2 k+l}\right|}\right|,
$$

where $\delta\left|\lambda_{2 k}\right|-1<r(k)<\delta\left|\lambda_{2 k}\right|+1$. Let $e^{-\varepsilon(k) k}<1 / 2$. By the definition of $\lambda_{k}$, we have $\left|\lambda_{2 k}-\lambda_{2 k-1}\right|=e^{-\varepsilon(k) k},\left|\lambda_{2 k}-\lambda_{2 k+l}\right|=m$ if $l=2 m$, and $\left|\lambda_{2 k}-\lambda_{2 k+l}\right| \geq m-1 / 2 \geq m / 2$ if $l=2 m-1$. Consequently,

$$
\ln \left|q_{\Lambda}^{2 k}\left(\lambda_{2 k}, \delta\right)\right| \geq-\varepsilon(k) k+\ln \frac{(r(k) !)^{4}}{4^{r(k)}(3 \delta((1+\delta) k+2))^{4 r(k)}} .
$$

Here we have also used the estimate $\left|\lambda_{2 k+l}\right| \leq(1+\delta)\left|\lambda_{2 k}\right|+2=(1+\delta) k+2$, which is valid because all $\lambda_{2 k+l}$ involved belong to the disk $B\left(\lambda_{2 k}, \delta\left|\lambda_{2 k}\right|+2\right)$. Since $k ! \geq 3^{-k} k^{k}$ for all $k=1,2, \ldots$, and $\delta \in(0,1 / 3)$, we obtain

$$
\ln \left|q_{\Lambda}^{2 k}\left(\lambda_{2 k}, \delta\right)\right| \geq-\varepsilon(k)+4 r(k) \ln \frac{r(k)}{3 \sqrt{2}(3 \delta((1+\delta) k+2))} \geq-\varepsilon(k) k+4 r(k) \ln \frac{r(k)}{12(k+1)} .
$$

Since $\delta\left|\lambda_{2 k}\right|-1<r(k)<\delta\left|\lambda_{2 k}\right|+1$, the value of the logarithm on the right in the last inequality is negative. Therefore,

$$
\begin{aligned}
\frac{\ln \left|q_{\Lambda}^{2 k}\left(\lambda_{2 k}, \delta\right)\right|}{\left|\lambda_{2 k}\right|}=\frac{\ln \left|q_{\Lambda}^{2 k}\left(\lambda_{2 k}, \delta\right)\right|}{k} & \geq-\varepsilon(k)+\frac{4\left(\delta\left|\lambda_{2 k}\right|+1\right)}{k} \ln \frac{\delta\left|\lambda_{2 k}\right|-1}{12(k+1)} \\
& =-\varepsilon(k)+\frac{4\left(\delta_{k}+1\right)}{k} \ln \frac{\delta_{k}-1}{12(k+1)} .
\end{aligned}
$$

Similarly, we obtain

$$
\frac{\ln \left|q_{\Lambda}^{2 k-1}\left(\lambda_{2 k-1}, \delta\right)\right|}{\left|\lambda_{2 k-1}\right|} \geq-\frac{\varepsilon(k) k}{k-e^{-\varepsilon(k) k}}+\frac{4(\delta k+1)}{k-e^{-\varepsilon(k) k}} \ln \frac{\delta_{k}-1}{12(k+1)} .
$$

Thus, by the choice of $\varepsilon(k)$, we have

$$
S_{\Lambda}=\lim _{\delta \rightarrow 0} \liminf _{k \rightarrow \infty} \frac{\ln \left|q_{\Lambda}^{k}\left(\lambda_{k}, \delta\right)\right|}{\left|\lambda_{k}\right|} \geq \lim _{\delta \rightarrow 0} 4 \delta \ln \frac{\delta}{12}=0 .
$$

We present yet another example to illustrate the quantity $S_{\Lambda}$. By the way, this example will be required later on. Let $\Lambda=\left\{\lambda_{k}\right\}$, where the $\lambda_{k}$ are positive numbers such that $\lambda_{k+1}-\lambda_{k} \geq h$. We calculate $S_{\Lambda}$. We have

$$
\ln \left|q_{\Lambda}^{k}\left(\lambda_{k}, \delta\right)\right|=\sum_{1 \leq l \leq s(k)} \ln \left|\frac{\lambda_{k}-\lambda_{k-l}}{3 \delta\left|\lambda_{k-l}\right|}\right|+\sum_{1 \leq l \leq p(k)} \ln \left|\frac{\lambda_{k}-\lambda_{k+l}}{3 \delta\left|\lambda_{k+l}\right|}\right|,
$$

where $s(k)$ is the number of the points $\lambda_{l}$ that belong to $B\left(\lambda_{k}, \delta\left|\lambda_{k}\right|\right)$ and lie to the right of $\lambda_{k}$. By construction, $\left|\lambda_{k}-\lambda_{k+l}\right| \geq l h$. Consequently, for all $\delta \in(0,1 / 3)$ we have

$$
\begin{aligned}
\ln \left|q_{\Lambda}^{k}\left(\lambda_{k}, \delta\right)\right| & \geq \ln \frac{h^{s(k)} s(k) !}{\left(3 \delta(1+\delta)\left|\lambda_{k}\right|\right)^{s(k)}}+\ln \frac{h^{p(k)} p(k) !}{\left(3 \delta(1+\delta)\left|\lambda_{k}\right|\right)^{p(k)}} \\
& \geq \ln \frac{h^{s(k)} s(k) !}{\left(6\left|\lambda_{k}\right|\right)^{s(k)}}+\ln \frac{h^{p(k)} p(k) !}{\left(6\left|\lambda_{k}\right|\right)^{p(k)}} \\
& \geq \ln \frac{h^{s(k)}(s(k))^{s(k)}}{\left(18\left|\lambda_{k}\right|\right)^{s(k)}}+\ln \frac{h^{p(k)}(p(k))^{p(k)}}{\left(18\left|\lambda_{k}\right|\right)^{p(k)}} \\
& \geq s(k) \ln \frac{h s(k)}{18\left|\lambda_{k}\right|}+p(k) \ln \frac{h p(k)}{18\left|\lambda_{k}\right|}
\end{aligned}
$$


Observe that the definitions of $s(k), p(k)$, and $\lambda_{l}$ imply

$$
\frac{s(k)}{\left|\lambda_{k}\right|} \leq \frac{M_{\Lambda}\left(\lambda_{k}, \delta\right)}{\left|\lambda_{k}\right|} \leq \frac{2 \delta\left|\lambda_{k}\right|}{h\left|\lambda_{k}\right|}=\frac{2 \delta}{h}, \quad \frac{p(k)}{\left|\lambda_{k}\right|} \leq \frac{M_{\Lambda}\left(\lambda_{k}, \delta\right)}{\left|\lambda_{k}\right|} \leq \frac{2 \delta}{h} .
$$

Next, the function $x \ln (h x / 18)$ is monotone decreasing when $h x / 18<1$. Therefore, taking the preceding inequalities into account, we obtain

$$
\begin{aligned}
S_{\Lambda}=\lim _{\delta \rightarrow 0} \liminf _{k \rightarrow \infty} \frac{\ln \left|q_{\Lambda}^{k}\left(\lambda_{k}, \delta\right)\right|}{\left|\lambda_{k}\right|} & \geq \lim _{\delta \rightarrow 0} \liminf _{k \rightarrow \infty}\left(\frac{s(k)}{\left|\lambda_{k}\right|} \ln \frac{h s(k)}{18\left|\lambda_{k}\right|}+\frac{p(k)}{\left|\lambda_{k}\right|} \ln \frac{h p(k)}{18\left|\lambda_{k}\right|}\right) \\
& \geq \lim _{\delta \rightarrow 0}\left(\frac{2 \delta}{h} \ln \frac{2 \delta}{18}+\frac{2 \delta}{h} \ln \frac{2 \delta}{18}\right)=0 .
\end{aligned}
$$

Now, we explore the relationship between the characteristics of the sequence $\Lambda$ introduced above. First, we observe that $M_{\Lambda}=0$ in all examples discussed. At the same time, $S_{\Lambda}$ may be negative and may be equal to zero. The situation changes if $M_{\Lambda}>0$.

Lemma 2.1. Let $\delta \in(0,1)$. We have

$$
\ln \left|q_{\Lambda}^{k}\left(\lambda_{k}, \delta\right)\right| \leq \ln \frac{1}{3(1-\delta)}\left(M_{\Lambda}\left(\lambda_{k}, \delta\right)-m_{k}\right), \quad k=1,2, \ldots
$$

If the sequence $\Lambda=\left\{\lambda_{k}, m_{k}\right\}_{k=1}^{\infty}$ satisfies $m(\Lambda)=0$ and $M_{\Lambda} \geq \tau>0$, then $S_{\Lambda} \leq-\tau \ln 3$.

Proof. The definition of the function $q_{\Lambda}^{k}\left(\lambda_{k}, \delta\right)$ shows that

$$
\begin{aligned}
\ln \left|q_{\Lambda}^{k}\left(\lambda_{k}, \delta\right)\right| & =\sum_{\substack{\lambda_{l} \in B\left(\lambda_{k}, \delta\left|\lambda_{k}\right|\right) \\
\lambda_{l} \neq \lambda_{k}}} m_{l} \ln \left|\frac{\lambda_{k}-\lambda_{l}}{3 \delta\left|\lambda_{l}\right|}\right| \leq \sum_{\substack{\lambda_{l} \in B\left(\lambda_{k}, \delta\left|\lambda_{k}\right|\right) \\
\lambda_{l} \neq \lambda_{k}}} m_{l} \ln \frac{\delta\left|\lambda_{k}\right|}{3 \delta(1-\delta)\left|\lambda_{k}\right|} \\
& =\ln \frac{1}{3(1-\delta)} \sum_{\substack{\lambda_{l} \in B\left(\lambda_{k}, \delta\left|\lambda_{k}\right|\right) \\
\lambda_{l} \neq \lambda_{k}}} m_{l}=\ln \frac{1}{3(1-\delta)}\left(M_{\Lambda}\left(\lambda_{k}, \delta\right)-m_{k}\right) .
\end{aligned}
$$

Suppose that $M_{\Lambda} \geq \tau>0$ and $m(\Lambda)=0$. Since the coefficient $-\ln (3(1-\delta))$ is negative for small $\delta>0$, the above shows that

$$
\begin{aligned}
S_{\Lambda}=\lim _{\delta \rightarrow 0} \liminf _{k \rightarrow \infty} \frac{\ln \left|q_{\Lambda}^{k}\left(\lambda_{k}, \delta\right)\right|}{\left|\lambda_{k}\right|} & \leq \lim _{\delta \rightarrow 0} \ln \frac{1}{3(1-\delta)} \limsup _{k \rightarrow \infty} \frac{\left(M_{\Lambda}\left(\lambda_{k}, \delta\right)-m_{k}\right)}{\left|\lambda_{k}\right|} \\
& =-\ln 3 \lim _{\delta \rightarrow 0} \limsup _{k \rightarrow \infty} \frac{M_{\Lambda}\left(\lambda_{k}, \delta\right)}{\left|\lambda_{k}\right|}=-\tau \ln 3 .
\end{aligned}
$$

The lemma is proved.

Let $\Gamma$ be an angle with vertex at the origin. We introduce the quantities

$$
N(\Lambda, \Gamma, t)=\sum_{\lambda_{k} \in \Gamma \cap B(0, t)} m_{k}, \quad N(\Lambda, \Gamma)=\limsup _{t \rightarrow \infty} \frac{N(\Lambda, \Gamma, t)}{t} .
$$

It can easily be observed that $N(\Lambda, \mathbb{C})=N(\Lambda)$. For $\xi \in \mathbb{S}$ (S is the unit circle with center at the origin) and $\delta \in(0,1)$, we denote by $\Gamma(\xi, \delta)$ the angle with vertex at the origin generated by the disk $B(\xi, \delta)$. Also, we put

$$
M_{\Lambda, \xi, \delta}=\limsup _{t \rightarrow \infty} \frac{M_{\Lambda}(t \xi, \delta)}{t} .
$$

Lemma 2.2. If $\xi \in \mathbb{S}, \delta \in(0,1)$, and the sequence $\Lambda=\left\{\lambda_{k}, m_{k}\right\}_{k=1}^{\infty}$ satisfies the condition $N(\Lambda, \Gamma(\xi, \delta))<\infty$, then

$$
\delta N(\Lambda, \Gamma(\xi, \delta)) \leq M_{\Lambda, \xi, 2 \delta} \leq(1+2 \delta) N(\Lambda, \Gamma(\xi, 2 \delta)) .
$$


Proof. First, we observe that the disk $B(t \xi, 2 t \delta)$ includes the part of the angle $\Gamma(\xi, \delta)$ that belongs to the annulus $B(0, t) \backslash B(0,(1-\delta) t)$. Indeed, by the definition of $\Gamma(\xi, \delta)$, for all $w \in \Gamma(\xi, \delta)$ with $(1-\delta) t \leq|w| \leq t$ we have

$$
|w-t \xi| \leq|w-| w|\xi|+|| w|\xi-t \xi| \leq|w| \delta+\delta t \leq 2 t \delta
$$

Consequently,

$$
\frac{N(\Lambda, \Gamma(\xi, \delta), t)}{t} \leq \frac{N(\Lambda, \Gamma(\xi, \delta),(1-\delta) t)}{t}+\frac{M_{\Lambda}(t \xi, 2 \delta)}{t}
$$

It follows that

$$
\begin{aligned}
N(\Lambda, \Gamma(\xi, \delta)) & =\limsup _{t \rightarrow \infty} \frac{N(\Lambda, \Gamma(\xi, \delta), t)}{t} \\
& \leq \limsup _{t \rightarrow \infty} \frac{N(\Lambda, \Gamma(\xi, \delta),(1-\delta) t)}{t}+\limsup _{t \rightarrow \infty} \frac{M_{\Lambda}(t \xi, 2 \delta)}{t} \\
& =(1-\delta) \limsup _{r \rightarrow \infty} \frac{N(\Lambda, \Gamma(\xi, \delta), r)}{r}+M_{\Lambda, \xi, 2 \delta} \\
& =(1-\delta) N(\Lambda, \Gamma(\xi, \delta))+M_{\Lambda, \xi, 2 \delta} .
\end{aligned}
$$

This yields the first inequality claimed. The second is a consequence of the estimate

$$
\frac{M_{\Lambda}(t \xi, 2 \delta)}{t} \leq \frac{N(\Lambda, \Gamma(\xi, \delta),(1+2 \delta) t)}{t}
$$

The lemma is proved.

\section{§3. Construction of a special function}

Along with the condensation index, the quantity $S_{\Lambda}$ influences the presence of singular points on the boundary of the convergence domain for the series of exponential monomials. If $S_{\Lambda}$ is negative, then there exists a sequence $d \in \mathfrak{A}(\Lambda)$ such that $g_{d}(z)$ has no singular points on the boundary of the set $D(\Lambda, d)$. We present a statement in which a function with this property is constructed. This function is a generalization of a special function in the theory of Dirichlet series (see [9]). We need some notation. For a convex domain $D$ and a subset $\Theta$ of the circle $\mathbb{S}$, we put

$$
D(\Theta)=\left\{z \in \mathbb{C}: \operatorname{Re}(z \lambda)<H_{D}(\lambda), \lambda \in \Theta\right\},
$$

where

$$
H_{M}(\lambda)=\sup _{z \in M} \operatorname{Re}(z \lambda)
$$

is the support function for $M$ (more precisely, for the complex conjugation of $M$ ). The set $D(\Theta)$ is convex, being an intersection of convex sets (specifically, hyperplanes). Since the support function of an arbitrary set is lower semicontinuous (see [16]), the set $D(\Theta)$ is a convex domain. The definitions of $D(\Theta)$ and $H_{D}(\lambda)$ readily imply the inclusion $D \subseteq D(\Theta)$. We denote by $\Theta(\Lambda)$ the set of limit points of the sequence $\left\{\lambda_{k} /\left|\lambda_{k}\right|\right\}$ (except the point $\lambda_{k}=0$ if it occurs). Clearly, $\Theta(\Lambda)$ is a convex subset of $\mathbb{S}$.

Theorem 3.1. Suppose a sequence $\lambda=\left\{\lambda_{k}, m_{k}\right\}_{k=1}^{\infty}$ is such that $m(\Lambda)=0$ and for some subsequence $\widetilde{\Lambda}=\left\{\lambda_{k(p)}\right\}_{p=1}^{\infty}$ we have the inequality

$$
\limsup _{p \rightarrow \infty} \frac{\ln \left|q_{\Lambda}^{k(p)}\left(\lambda_{k(p)}, \delta_{p}\right)\right|}{\left|\lambda_{k(p)}\right|} \leq-\beta<0,
$$

where $\left\{\delta_{p}\right\}$ is a sequence on the interval $(0,1 / 4)$ monotone decreasing to zero. Then for every bounded convex domain $D$ there exists a sequence $d \in \mathfrak{A}(\Lambda)$ such that the set $D(\Lambda, d)$ coincides with $D(\Theta(\widetilde{\Lambda}))$ and the function $g_{d}(z)$ is analytic in $D_{\beta}=D(\Lambda, d)+B(0, \beta)$. 
Remark. If $S_{\Lambda} \leq-\beta$ then, by the definition of $S_{\Lambda}$, there exists a subsequence $\left\{\lambda_{k(p)}\right\}$ of $\left\{\lambda_{k}\right\}$ satisfying (3.1).

Proof. We shall look for the required function in the form

$$
g(z)=\sum_{p=1}^{\infty} c_{p} g_{p}(z) .
$$

To begin with, we "rarefy" the sequence $\Lambda=\left\{\lambda_{k}, m_{k}\right\}_{k=1}^{\infty}$ duly, i.e., from elements of $\Lambda$ we construct a new sequence with special properties. To lighten the notation, we use the same symbols for the new sequence as for the initial one.

We proceed to extracting the subsequence. We start with the observation that, passing to a subsequence, we may assume that

$$
\left|\lambda_{k(p+1)}\right| \geq 2\left|\lambda_{k(p)}\right|, \quad p=1,2, \ldots
$$

Moreover, this can be achieved without reducing the set $\Theta(\widetilde{\Lambda})$. To ensure this, we employ some countable dense subset of $\Theta(\widetilde{\Lambda})$ when taking the subsequence. Next, we remove all "irrelevant" points from $\Lambda$. At the beginning, we discard all $\lambda_{k}$ that do not belong to any of the disks $B_{p}=B\left(\lambda_{k(p)}, \delta_{p}\left|\lambda_{k(p)}\right|\right), p=1,2, \ldots$ Next, if $p$ satisfies

$$
M_{\Lambda}\left(\lambda_{k(p)}, \delta_{p}\right)-m_{k(p)} \geq \beta\left|\lambda_{k(p)}\right|+1,
$$

then, without touching $\lambda_{k(p)}$, we remove some of $\lambda_{k}$ 's from $B_{p}$ (or reduce their multiplicities $m_{k}$ ) so that the following inequalities be fulfilled:

$$
\beta\left|\lambda_{k(p)}\right| \leq M_{\Lambda}\left(\lambda_{k(p)}, \delta_{p}\right)-m_{k(p)}<\beta\left|\lambda_{k(p)}\right|+1 .
$$

This leads to a sequence $\Lambda=\left\{\lambda_{k}, m_{k}\right\}_{k=1}^{\infty}$ satisfying the following conditions:

1) each point $\lambda_{k}$ belongs to precisely one disk among the $B_{p}, p=1,2, \ldots$;

2) the series $\sum_{p=1}^{\infty} \exp \left(-\alpha\left|\lambda_{k(p)}\right|\right)$ converges for every $\alpha>0$;

$3)$ inequality (3.1) holds true;

4) $m(\Lambda)=0$

5) $N(\Lambda)<\infty$.

We show that conditions 1)-5) are satisfied indeed. By construction, each $\lambda_{k}$ belongs to some $B_{p}, p=1,2, \ldots$ Next, (3.3) is true. Since $\delta_{p}<1 / 4,(3.3)$ shows that

$$
\left(1+\delta_{p}\right)\left|\lambda_{k(p)}\right| \leq\left(1-\delta_{p+1}\right)\left|\lambda_{k(p+1)}\right|, \quad p=1,2, \ldots
$$

Therefore, the disks $B_{p}, p=1,2, \ldots$, are mutually disjoint, and 1) follows. Property 2 ) is an immediate consequence of (3.3). We prove 3). If (3.4) fails for a particular $p$, then all points $\lambda_{k}$ in $B_{p}$ have remained where they were, and the quantity $\ln \left|q_{\Lambda}^{k(p)}\left(\lambda_{k(p)}, \delta_{p}\right)\right|$ has not changed. Let $p$ be an index satisfying (3.4). After discarding some $\lambda_{k}$ 's belonging to $B_{p}$ (or after reducing their multiplicities $\lambda_{k}$ ), the quantity $\ln \left|q_{\Lambda}^{k(p)}\left(\lambda_{k(p)}, \delta_{p}\right)\right|$ increases, because every factor in the formula defining $q_{\Lambda}^{k(p)}\left(\lambda_{k(p)}, \delta_{p}\right)$ does not exceed 1 in modulus. However, this increase cannot lead to the violation of the required upper estimates. Indeed, by Lemma 2.1 we have

$$
\ln \left|q_{\Lambda}^{k(p)}\left(\lambda_{k(p)}, \delta_{p}\right)\right| \leq \ln \frac{1}{3\left(1-\delta_{p}\right)}\left(M_{\Lambda}\left(\lambda_{k(p)}, \delta_{p}\right)-m_{k(p)}\right) .
$$

By this formula and (3.5), for large $p$ we obtain

$$
\ln \left|q_{\Lambda}^{k(p)}\left(\lambda_{k(p)}, \delta_{p}\right)\right| \leq-\left(M_{\Lambda}\left(\lambda_{k(p)}, \delta_{p}\right)-m_{k(p)}\right) \leq-\beta\left|\lambda_{k(p)}\right| .
$$

So, inequality (3.1) is still fulfilled for the new sequence. 
Condition 4) is satisfied because the limit of a subsequence coincides with the limit of the original sequence, and the relation $m(\Lambda)=0$ remains true if we reduce some of the $m_{k}>0$. We verify condition 5$)$. By construction, for every $p=1,2, \ldots$ we have

$$
M_{\Lambda}\left(\lambda_{k(p)}, \delta_{p}\right)-m_{k(p)}<\beta\left|\lambda_{k(p)}\right|+1 .
$$

Let $t \in\left[\left(1+\delta_{p}\right)\left|\lambda_{k(p)}\right|,\left(1-\delta_{p+1}\right)\left|\lambda_{k(p+1)}\right|\right]$. By (3.6) and condition 1), we obtain

$$
\begin{aligned}
t^{-1} N(\Lambda, \mathbb{C}, t) & =t^{-1} N\left(\Lambda, \mathbb{C},\left(1+\delta_{p}\right)\left|\lambda_{k(p)}\right|\right)=t^{-1} \sum_{j=1}^{p} M_{\Lambda}\left(\lambda_{k(j)}, \delta_{j}\right) \\
& \leq\left(\left(1+\delta_{p}\right)\left|\lambda_{k(p)}\right|\right)^{-1} \sum_{j=1}^{p} M_{\Lambda}\left(\lambda_{k(j)}, \delta_{j}\right) .
\end{aligned}
$$

By (3.3) and (3.7), it follows that

$$
\begin{aligned}
t^{-1} N(\Lambda, \mathbb{C}, t) & \leq\left(\left(1+\delta_{p}\right)\left|\lambda_{k(p)}\right|\right)^{-1} \sum_{j=1}^{p}\left(\beta\left|\lambda_{k(j)}\right|+1+m_{k(j)}\right) \\
& \leq\left|\lambda_{k(p)}\right|^{-1} \sum_{j=1}^{p}\left(2^{j-p} \beta\left|\lambda_{k(p)}\right|+1\right)+\sum_{j=1}^{p} 2^{j-p}\left|\lambda_{k(j)}\right|^{-1} m_{k(j)} .
\end{aligned}
$$

By (3.3), the sequence $\left\{p\left|\lambda_{k(p)}\right|^{-1}\right\}$ is bounded. Condition 4) shows that the sequence $\left\{\left|\lambda_{k(j)}\right|^{-1} m_{k(j)}\right\}$ is also bounded. Thus, by the above, we obtain

$$
\begin{aligned}
t^{-1} N(\Lambda, \mathbb{C}, t) & \leq \sum_{j=1}^{p}\left(2^{j-p} \beta+\left|\lambda_{k(p)}\right|^{-1}\right)+\sum_{j=1}^{p} 2^{j-p}\left|\lambda_{k(j)}\right|^{-1} m_{k(j)} \\
& \leq p\left|\lambda_{k(p)}\right|^{-1}+\beta \sum_{j=1}^{p} 2^{j-p}+c_{1} \sum_{j=1}^{p} 2^{j-p} \leq c_{2}+\beta+c_{1} .
\end{aligned}
$$

Now, let $t \in\left[\left(1-\delta_{p}\right)\left|\lambda_{k(p)}\right|,\left(1+\delta_{p}\right)\left|\lambda_{k(p)}\right|\right]$. Then

$$
\begin{aligned}
\frac{N(\Lambda, \mathbb{C}, t)}{t} & \leq \frac{N\left(\Lambda, \mathbb{C},\left(1+\delta_{p}\right)\left|\lambda_{k(p)}\right|\right)}{t} \leq \frac{N\left(\Lambda, \mathbb{C},\left(1+\delta_{p}\right)\left|\lambda_{k(p)}\right|\right)}{\left(1-\delta_{p}\right)\left|\lambda_{k(p)}\right|} \\
& \leq 2\left|\lambda_{k(p)}\right|^{-1} N\left(\Lambda, \mathbb{C},\left(1+\delta_{p}\right)\left|\lambda_{k(p)}\right|\right) \leq 2\left(c_{2}+\beta+c_{1}\right) .
\end{aligned}
$$

So, $N(\Lambda)=N(\Lambda, \mathbb{C})<\infty$.

Now, we define functions $g_{p}(z), p=1,2, \ldots$, by the formula

$$
g_{p}(z)=\frac{1}{2 \pi i} \int_{S\left(\lambda_{k(p)}, 4 \delta_{p}\left|\lambda_{k(p)}\right|\right)} \frac{\exp (\lambda z) d \lambda}{a_{p}\left(\lambda-\lambda_{k(p)}\right) q_{\Lambda}^{k(p)}\left(\lambda, \delta_{p}\right)},
$$

where $S(b, r)$ is the circle of radius $r$ and with center $b$, and the quantities $a_{p} \geq 1$ will be specified later. We estimate these functions from above. Since $a_{p} \geq 1$, we have

$$
\begin{aligned}
\left|a_{p} q_{\Lambda}^{k(p)}\left(\lambda, \delta_{p}\right)\right| & \geq\left|q_{\Lambda}^{k(p)}\left(\lambda, \delta_{p}\right)\right|=\left|\prod_{\substack{\lambda_{k} \in B\left(\lambda_{k(p)}, \delta_{p}\left|\lambda_{k(p)}\right|\right) \\
\lambda_{k} \neq \lambda_{k(p)}}}\left(\frac{\lambda-\lambda_{k}}{3 \delta_{p}\left|\lambda_{k}\right|}\right)^{m_{k}}\right| \\
& \geq\left|\prod_{\substack{\lambda_{k} \in B\left(\lambda_{k(p)}, \delta_{p}\left|\lambda_{k(p)}\right|\right) \\
\lambda_{k} \neq \lambda_{k(p)}}}\left(\frac{3 \delta_{p}\left|\lambda_{k}\right|}{3 \delta_{p}\left|\lambda_{k}\right|}\right)^{m_{k}}\right|=1, \\
& \lambda \in S\left(\lambda_{k(p)}, 4 \delta_{p}\left|\lambda_{k(p)}\right|\right) .
\end{aligned}
$$


Consequently,

$$
\begin{aligned}
\left|g_{p}(z)\right| & =\left|\frac{1}{2 \pi i} \int_{S\left(\lambda_{k(p)}, 4 \delta_{p}\left|\lambda_{k(p)}\right|\right)} \frac{\exp (\lambda z) d \lambda}{a_{p}\left(\lambda-\lambda_{k(p)}\right) q_{\Lambda}^{k(p)}\left(\lambda, \delta_{p}\right)}\right| \\
& \leq \frac{8 \pi\left|\lambda_{k(p)}\right| \delta_{p}}{2 \pi} \sup _{\lambda \in S\left(\lambda_{k(p)}, 4 \delta_{p}\left|\lambda_{k(p)}\right|\right)}\left|\frac{\exp (\lambda z)}{a_{p}\left(\lambda-\lambda_{k(p)}\right) q_{\Lambda}^{k(p)}\left(\lambda, \delta_{p}\right)}\right| \\
& \leq 4 \pi\left|\lambda_{k(p)}\right| \delta_{p} \sup _{\lambda \in S\left(\lambda_{k(p)}, 4 \delta_{p}\left|\lambda_{k(p)}\right|\right)}\left|\frac{\exp (\lambda z)}{\left(\lambda-\lambda_{k(p)}\right)}\right| \\
& \leq \sup _{\lambda \in S\left(\lambda_{k(p)}, 4 \delta_{p}\left|\lambda_{k(p)}\right|\right)}|\exp (\lambda z)| \\
& \leq \exp \left(\operatorname{Re}\left(\lambda_{k(p)} z\right)+4 \delta_{p}\left|\lambda_{k(p)}\right||z|\right), \quad z \in \mathbb{C} .
\end{aligned}
$$

Now, we define the coefficients $c_{p}$. Let $D$ be a bounded convex domain, and let $\left\{K_{l}\right\}_{l=1}^{\infty}$ be a strictly exhausting sequence of compact convex sets for $D$ (i.e., for every $l=1,2, \ldots$ the interior of $K_{l+1}$ includes $K_{l}$, and $D$ is the union of all $K_{l}$ ). Put

$$
c_{p}=\exp \left(-H_{K_{p}}\left(\lambda_{k(p)}\right)-\beta\left|\lambda_{k(p)}\right|\right), \quad p=1,2, \ldots
$$

We find the domain of convergence of the series (3.2). Set

$$
\widetilde{D}=\left\{z \in \mathbb{C}: \operatorname{Re}(z \lambda)<H_{D}(\lambda)+\beta, \lambda \in \Theta(\widetilde{\Lambda})\right\} .
$$

We show that the series (3.2) converges uniformly on compact subset of $\widetilde{D}$. Fixing a compact set $K \subset \widetilde{D}$, we take $z \in K$ and $\lambda \in \Theta(\widetilde{\Lambda})$. By the definition of $\widetilde{D}$, we have $\operatorname{Re}(z \lambda)<H_{D}(\lambda)+\beta$. Since $\left\{K_{l}\right\}_{l=1}^{\infty}$ is an exhausting sequence for $D$, we have $H_{K_{l}}(\lambda) \rightarrow H_{D}(\lambda)$ as $l \rightarrow \infty$, by the definition of the support function. Therefore, there exists an index $l(z, \lambda)$ such that $\operatorname{Re}(z \lambda)<H_{K_{l(z, \lambda)}}(\lambda)+\beta$. Since the support function of a compact set is continuous (see [16]), it follows that the last inequality is valid in some neighborhoods $U(z)$ and $V(\lambda)$ of $z$ and $\lambda$, respectively:

$$
\operatorname{Re}(w \xi)<H_{K_{l(z, \lambda)}}(\xi)+\beta, \quad w \in U(z), \quad \xi \in V(\lambda) .
$$

The sets $U(z) \times V(\lambda), z \in K, \lambda \in \Theta$, cover the compact set $K \times \Theta$, allowing us to extract a finite subcovering $U(z(j)) \times V(\lambda(j)), j=1, \ldots, s$. Let $r$ be the greatest among the indices $l(z(j), \lambda(j)), j=1, \ldots, s$. The union of the sets $U(z(j)) \times V(\lambda(j)), j=1, \ldots, s$, includes a set of the form $K \times V$, where $V$ is a neighborhood of the compact set $\Theta$. Let $z \in K, \lambda \in V$. By (3.9), for some $j=1, \ldots, s$ we have $\operatorname{Re}(z \lambda)<H_{K_{l(z(j), \lambda(j))}}(\lambda)$. Since $\left\{K_{l}\right\}$ is a monotone increasing sequence of sets, we have

$$
H_{K_{l(z(j), \lambda(j))}}(\lambda) \leq H_{K_{r}}(\lambda) .
$$

Thus, we have obtained the estimate

$$
\operatorname{Re}(z \lambda)<H_{K_{r}}+\beta, \quad z \in K, \lambda \in V .
$$

Using the definition of $\Theta(\widetilde{\Lambda})$, we choose an index $p_{0}$ such that $\lambda_{k(p)} /\left|\lambda_{k(p)}\right|$ belongs to $V$ for all $p \geq p_{0}$. Since a support function is positive homogeneous of order 1 , from (3.8) and (3.10) we deduce that

$$
\begin{aligned}
\left|g_{p}(z)\right| & \leq \exp \left(\left|\lambda_{k(p)}\right| \operatorname{Re}\left(\frac{\lambda_{k(p)} z}{\left|\lambda_{k(p)}\right|}\right)+4 \delta_{p}\left|\lambda_{k(p)}\right||z|\right) \\
& <\exp \left(\left|\lambda_{k(p)}\right|\left(H_{K_{r}}\left(\frac{\lambda_{k(p)}}{\left|\lambda_{k(p)}\right|}\right)+\beta\right)+4 \delta_{p}\left|\lambda_{k(p)}\right||z|\right) \\
& =\exp \left(H_{K_{r}}\left(\lambda_{k(p)}\right)+\beta\left|\lambda_{k(p)}\right|+4 \delta_{p}\left|\lambda_{k(p)}\right||z|\right) .
\end{aligned}
$$


Since $K_{r}$ lies in the interior of $K_{r+1}$ and the sequence $\left\{K_{l}\right\}_{l=1}^{\infty}$ is monotone increasing, there is $\alpha_{r}>0$ with

$$
H_{K_{r}}(\lambda)+2 \alpha_{r}|\lambda| \leq H_{K_{r+1}}(\lambda) \leq H_{K_{l}}(\lambda), \quad \lambda \in \mathbb{C}, \quad l \geq r+1 .
$$

We choose $p_{1} \geq \max \left\{p_{0}, r+1\right\}$ such that $4 \delta_{p}|z| \leq \alpha_{r}$ for $z \in K$ and $p \geq p_{1}$. Then (3.11), (3.12), and the definition of the coefficients $c_{p}$ show that

$$
\begin{aligned}
\left|c_{p} g_{p}(z)\right| & \leq \exp \left(-H_{K_{p}}\left(\lambda_{k(p)}\right)-\beta\left|\lambda_{k(p)}\right|\right) \exp \left(H_{K_{r}}\left(\lambda_{k(p)}\right)+\beta\left|\lambda_{k(p)}\right|+4 \delta_{p}\left|\lambda_{k(p)}\right||z|\right) \\
& \leq \exp \left(-2 \alpha_{r}\left|\lambda_{k(p)}\right|+4 \delta_{p}\left|\lambda_{k(p)}\right||z|\right) \\
& \leq \exp \left(-2 \alpha_{r}\left|\lambda_{k(p)}\right|+\alpha_{r}\left|\lambda_{k(p)}\right|\right)=\exp \left(-\alpha_{r}\left|\lambda_{k(p)}\right|\right)
\end{aligned}
$$

for all $z \in K$ and $p \geq p_{1}$. Therefore,

$$
\sum_{p=p_{1}}^{\infty}\left|c_{p} g_{p}(z)\right| \leq \sum_{p=p_{1}}^{\infty} \exp \left(-\alpha_{r}\left|\lambda_{k(p)}\right|\right), \quad z \in K .
$$

Property 2) of the sequence $\Lambda$ implies that the last series converges and, with it, the series (3.2) also converges, and even uniformly on compact subsets of $\widetilde{D}$. It should be noted that the above arguments are valid for any choice of $a_{p} \geq 1, p=1,2, \ldots$.

Now we show that, for a suitable choice of $a_{p} \geq 1, p=1,2, \ldots$, the function $g(z)$ expands in a series of the form (1.1) in some subdomain of $\widetilde{D}$. By the residue calculus, for every $p=1,2, \ldots$ we have

$$
g_{p}(z)=a_{p}^{-1}\left(b_{k(p), 0} \exp \left(\lambda_{k(p)} z\right)+\sum_{\substack{\lambda_{k} \in B\left(\lambda_{k(p)}, \delta_{p}\left|\lambda_{k(p)}\right|\right) \\ \lambda_{k} \neq \lambda_{k(p)}}} \sum_{n=0}^{m_{k}-1} b_{k, n} z^{n} \exp \left(\lambda_{k} z\right)\right),
$$

where $b_{k(p), 0}=\left(q_{\Lambda}^{k(p)}\left(\lambda_{k(p)}, \delta_{p}\right)\right)^{-1}$. We also put $b_{k(p), n}=0, n=1, \ldots, m_{k(p)}-1$. We define the coefficients $d_{k, n}$ as follows. Let $\lambda_{k} \in B_{p}$ for some $p=1,2, \ldots$ Then

$$
d_{k, n}=c_{p} b_{k, n}\left(a_{p}\right)^{-1}, \quad n=0,1, \ldots, m_{k}-1 .
$$

By property 1 ) of the sequence $\Lambda$, this definition is consistent.

Now we choose the numbers $a_{p}, p=1,2, \ldots$ By property 3 ),

$$
\liminf _{p \rightarrow \infty} Y_{p} \geq \beta
$$

where $Y_{p}=\max \left\{\left|\lambda_{k(p)}\right|^{-1} \ln \left|b_{k, n}\right|\right\}$ and the maximum is taken over all $k$ with $\lambda_{k} \in B_{p}$ and all $n=0,1, \ldots, m_{k}-1$. If $Y_{p} \leq \beta$, we put $a_{p}=1$, otherwise we choose $a_{p} \geq 1$ with $Y_{p}-\left|\lambda_{k(p)}\right|^{-1} \ln a_{p}=\beta$. Thus, we have

$$
\lim _{p \rightarrow \infty} \max _{\substack{k: \lambda_{k} \in B_{p} \\ 0 \leq n \leq m_{k}-1}}\left\{\left|\lambda_{k(p)}\right|^{-1} \ln \left|d_{k, n} / c_{p}\right|\right\}=\beta .
$$

We find the domain of convergence for the series

$$
\sum_{k=1, n=0}^{\infty, m_{k}-1} d_{k, n} z^{n} \exp \left(\lambda_{k} z\right)
$$

Property 5) shows that $\sigma(\Lambda)=0$. Then, by property 4) and the Cauchy-Hadamard theorem for series of exponential monomials, the series (3.14) converges in the convex domain

$$
D(\Lambda, d)=\{z \in \mathbb{C}: \operatorname{Re}(z \lambda)<h(d, \lambda), \lambda \in \Theta(\Lambda)\}
$$


and diverges at any point of its exterior, with a possible exception of the origin. The function $h(d, \lambda)$ is defined by the formula (see [2])

$$
h(d, \lambda)=\inf \liminf _{l \rightarrow \infty} \min _{0 \leq n \leq m_{s(l)}-1} \frac{\ln \left(1 /\left|d_{s(l), n}\right|\right)}{\left|\lambda_{l_{s}}\right|}, \quad \lambda \in \Theta(\Lambda),
$$

where the infimum is taken over all subsequences $\left\{\lambda_{s(l)}\right\}$ of the sequence $\left\{\lambda_{k}\right\}$ such that $\lambda_{s(l)} /\left|\lambda_{s(l)}\right|$ tends to $\lambda$ as $l \rightarrow \infty$.

We want to calculate the quantity $h(d, \lambda), \lambda \in \Theta(\Lambda)$. But before we show that $\Theta(\Lambda)=$ $\Theta(\tilde{\Lambda})$. The inclusion $\Theta(\tilde{\Lambda}) \subset \Theta(\Lambda)$ follows directly from the definitions. Let $\lambda \in \Theta(\Lambda)$. Then there exists a subsequence $\left\{\lambda_{s(l)}\right\}$ of $\left\{\lambda_{k}\right\}$ with $\lambda_{s(l)} /\left|\lambda_{s(l)}\right| \rightarrow \lambda$. By property 1$)$, every point $\lambda_{s(l)}$ belongs to some disk $B_{p(l)}$. Consequently, $\left|\lambda_{s(l)}-\lambda_{k(p(l))}\right|<\delta_{p(l)}\left|\lambda_{k(p(l))}\right|$. It follows that

$$
\begin{aligned}
\left|\frac{\lambda_{s(l)}}{\left|\lambda_{s(l)}\right|}-\frac{\lambda_{k(p(l))} \mid}{\left|\lambda_{k(p(l))}\right|}\right| & \leq\left|\frac{\lambda_{s(l)}}{\left|\lambda_{s(l)}\right|}-\frac{\lambda_{s(l)}}{\left|\lambda_{k(p(l))}\right|}\right|+\left|\frac{\lambda_{s(l)}}{\left|\lambda_{k(p(l))}\right|}-\frac{\lambda_{k(p(l))}}{\left|\lambda_{k(p(l))}\right|}\right| \\
& =\frac{|| \lambda_{k(p(l))}|-| \lambda_{s(l)} \mid}{\left|\lambda_{k(p(l))}\right|}+\frac{\left|\lambda_{k(p(l))}-\lambda_{s(l)}\right|}{\left|\lambda_{k(p(l))}\right|} \\
& \leq \delta_{p(l)}+\delta_{p(l)}=2 \delta_{p(l)} \rightarrow 0, \quad l \rightarrow \infty .
\end{aligned}
$$

This means that $\lambda \in \Theta(\widetilde{\Lambda})$, implying the required inclusion $\Theta(\Lambda) \subset \Theta(\widetilde{\Lambda})$.

Suppose $\lambda \in \Theta(\Lambda)$, a sequence $\left\{\lambda_{s(l)}\right\}$ has the property that $\lambda_{s(l)} /\left|\lambda_{s(l)}\right|$ tends to $\lambda$, and $\lambda_{s(l)} \in B_{p(l)}, l=1,2, \ldots$ We have

$$
\begin{aligned}
\min _{0 \leq n \leq m_{s(l)}-1} \frac{\ln \left(1 /\left|d_{s(l), n}\right|\right)}{\left|\lambda_{s(l)}\right|} & \geq \min _{\substack{k: \lambda_{k} \in B_{p(l)} \\
0 \leq n \leq m_{k}-1}} \frac{\ln \left(1 /\left|d_{k, n}\right|\right)}{\left|\lambda_{k}\right|} \\
& =-\max _{\substack{k: \lambda_{k} \in B_{p(l)} \\
0 \leq n \leq m_{k}-1}} \frac{\ln \left|d_{k, n}\right|}{\left|\lambda_{k}\right|}=-\max _{\substack{k: \lambda_{k} \in B_{p(l)} \\
0 \leq n \leq m_{k}-1}} \frac{\ln \left|d_{k, n}\right|}{\left|\lambda_{k(p(l))}\right|} \frac{\left|\lambda_{k(p(l))}\right|}{\left|\lambda_{k}\right|} \\
& \geq-\frac{1}{1 \pm \delta_{p(l)}} \underset{\substack{\begin{subarray}{c}{k: \lambda_{k} \in B_{p(l)} \\
0 \leq n \leq m_{k}-1} }} \\
{\left|\lambda_{k(p(l))}\right|}\end{subarray}}{ }
\end{aligned}
$$

Here the sign in the denominator is opposite to the sign of the maximum. By the definition of $c_{p}$, we obtain

$$
\begin{gathered}
\min _{0 \leq n \leq m_{s(l)}-1} \frac{\ln \left(1 /\left|d_{s(l), n}\right|\right)}{\left|\lambda_{s(l)}\right|} \geq-\frac{1}{1 \pm \delta_{p(l)}} \max _{\substack{k: \lambda_{k} \in B_{p(l)} \\
0 \leq n \leq m_{k}-1}} \frac{\ln \left|d_{k, n} / c_{p(l)}\right|+\ln \left|c_{p(l)}\right|}{\left|\lambda_{k(p(l))}\right|} \\
=-\frac{1}{1 \pm \delta_{p(l)}}\left(\max _{\substack{k: \lambda_{k} \in B_{p(l)} \\
0 \leq n \leq m_{k}-1}} \frac{\ln \left|d_{k, n} / c_{p(l)}\right|}{\left|\lambda_{k(p(l))}\right|}-H_{K_{p(l)}}\left(\frac{\lambda_{k(p(l))}}{\left|\lambda_{k(p(l))}\right|}\right)-\beta\right) .
\end{gathered}
$$

The sequence $\lambda_{k(p(l))} /\left|\lambda_{k(p(l))}\right|$ tends to $\lambda$ along with $\lambda_{s(l)} /\left|\lambda_{s(l)}\right|$, the sequence $\left\{K_{r}\right\}$ is monotone increasing, and the support function of a compact set is continuous. Consequently,

$$
\liminf _{l \rightarrow \infty} H_{K_{p(l)}}\left(\frac{\lambda_{k(p(l))}}{\left|\lambda_{k(p(l))}\right|}\right) \geq \liminf _{l \rightarrow \infty} H_{K_{r}}\left(\frac{\lambda_{k(p(l))}}{\left|\lambda_{k(p(l))}\right|}\right)=H_{K_{r}}(\lambda), \quad r=1,2, \ldots
$$

Next, the sequence $\left\{K_{r}\right\}$ exhausts the domain $D$. Therefore,

$$
\liminf _{l \rightarrow \infty} H_{K_{p(l)}}\left(\frac{\lambda_{k(p(l))}}{\left|\lambda_{k(p(l))}\right|}\right) \geq \lim _{r \rightarrow \infty} H_{K_{r}}(\lambda)=H_{D}(\lambda) .
$$


By (3.13) and (3.15), we obtain

$$
\begin{aligned}
\liminf _{l \rightarrow \infty} \min _{0 \leq n \leq m_{s(l)}-1} \frac{\ln \left(1 /\left|d_{s(l), n}\right|\right)}{\left|\lambda_{s(l)}\right|} \geq-\lim _{p \rightarrow \infty} \frac{1}{1 \pm \delta_{p(l)}} \max _{\substack{k: \lambda_{k} \in B_{p(l)} \\
0 \leq n \leq m_{k}-1}} \frac{\ln \left|d_{k, n} / c_{p(l)}\right|}{\left|\lambda_{k(p(l))}\right|} \\
+\liminf _{l \rightarrow \infty} H_{K_{p(l)}}\left(\frac{\lambda_{k(p(l))}}{\left|\lambda_{k(p(l))}\right|}\right)+\beta \geq-\beta+H_{D}(\lambda)+\beta=H_{D}(\lambda) .
\end{aligned}
$$

Since the sequence $\left\{\lambda_{s(l)}\right\}$ has been taken arbitrarily so as to ensure that $\lambda_{s(l)} /\left|\lambda_{s(l)}\right|$ tend to $\lambda$, the definition of $h(d, \lambda)$ implies

$$
h(d, \lambda) \geq H_{D}(\lambda), \quad \lambda \in \Theta(\Lambda)=\Theta(\widetilde{\Lambda}) .
$$

We claim that, in fact, equality occurs here. Suppose $\lambda \in \Theta(\widetilde{\Lambda})$ and a sequence $\left\{\lambda_{k(p(l))}\right\}$ is such that $\lambda_{k(p(l))} /\left|\lambda_{k(p(l))}\right|$ tends to $\lambda$. For every $p(l), l=1,2, \ldots$, we denote by $s(l)$ and $n(l)$ the indices $k$ and $n$ of the coefficient $d_{k, n}$ at which the maximum in (3.13) is attained. Then

$$
\lim _{l \rightarrow \infty}\left|\lambda_{k(p(l))}\right|^{-1} \ln \left|d_{s(l), n(l)} / c_{p(l)}\right|=\beta .
$$

It follows that

$$
\begin{aligned}
\limsup _{l \rightarrow \infty} \frac{\ln \left(1 /\left|d_{s(l), n(l)}\right|\right)}{\left|\lambda_{s(l)}\right|} & =\limsup _{l \rightarrow \infty} \frac{-\left(\ln \left|d_{s(l), n(l)} / c_{p(l)}\right|+\ln \left|c_{p(l)}\right|\right)\left|\lambda_{k(p(l))}\right|}{\left|\lambda_{s(l)}\right|\left|\lambda_{k(p(l))}\right|} \\
& =\limsup _{l \rightarrow \infty} \frac{-\left(\ln \left|d_{s(l), n(l)} / c_{p(l)}\right|+\ln \left|c_{p(l)}\right|\right)}{\left|\lambda_{k(p(l))}\right|} \\
& =-\beta+\limsup _{l \rightarrow \infty} \frac{\left.-\ln \left|c_{p(l)}\right|\right)}{\left|\lambda_{k(p(l))}\right|}=\lim _{l \rightarrow \infty} H_{K_{p(l)}}\left(\frac{\lambda_{k(p(l))}}{\left|\lambda_{k(p(l))}\right|}\right) .
\end{aligned}
$$

Since $K_{p(l)} \subset D$ and the function $H_{D}$ is continuous, we have

$$
\limsup _{l \rightarrow \infty} \frac{\ln \left(1 /\left|d_{s(l), n(l)}\right|\right)}{\left|\lambda_{s(l)}\right|} \leq \limsup _{l \rightarrow \infty} H_{D}\left(\frac{\lambda_{k(p(l))}}{\left|\lambda_{k(p(l))}\right|}\right)=H_{D}(\lambda) .
$$

Since $\lambda_{s(l)} \in B_{p(l)}$, as above we see that $\lambda_{s(l)} /\left|\lambda_{s(l)}\right|$ tends to $\lambda$. Then, by the definition of $h(d, \lambda)$, we have

$$
h(d, \lambda) \leq H_{D}(\lambda), \quad \lambda \in \Theta(\Lambda)=\Theta(\widetilde{\Lambda}) .
$$

Thus, we have proved the identity

$$
h(d, \lambda)=H_{D}(\lambda), \quad \lambda \in \Theta(\Lambda)=\Theta(\widetilde{\Lambda}) .
$$

Hence, by the definition of $D(\Lambda, d)$ and $D(\Lambda(\widetilde{\Lambda}))$, these two domains coincide. Suppose $z \in D_{\beta}$ and $\lambda \in \Theta(\Lambda)$. Then $z=w+y$, where $y \in B(0, \beta)$ and $w \in D(\Lambda, d)$. Together with the definitions of $D(\Lambda, d)$ and of the support function for the disk, (3.16) yields

$$
\operatorname{Re}(z \lambda)=\operatorname{Re}(w \lambda)+\operatorname{Re}(y \lambda)<H_{D}(\lambda)+\beta|\lambda|=H_{D}(\lambda)+\beta .
$$

Consequently, $D_{\beta} \subseteq \widetilde{D}$. It remains to show that $g_{d}(z)$ coincides with $g(z)$ in $D(\Lambda, d)$. Since $m(\Lambda)=\sigma(\Lambda)=0$, the Abel theorem for series of exponential monomials shows that the series (3.14) converges absolutely in $D(\Lambda, d)$. But the series (3.2) can be obtained from (3.14) by uniting terms into groups; therefore, on the joint domain of convergence $D(\Lambda, d)$, it has the same sum as (3.14). The theorem is proved. 


\section{$\S 4$. Singular POINTS}

By Theorem 3.1, under the condition $m(\Lambda)=0$, if $S_{\Lambda}$ is negative, then there exist analytic functions representable by a series of the form (3.14) with an arbitrarily large domain of convergence that have no singularities on the boundary of this domain. It turns out that, under a certain additional condition on $\Lambda$, the negativity of $S_{\Lambda}$ is necessary for the existence of such functions. We shall present the corresponding result in the sequel. But before that, we introduce still more notation and definitions, and also prove two auxiliary results.

A sequence $\Lambda=\left\{\lambda_{k}, m_{k}\right\}_{k=1}^{\infty}$ is said to be proper if it is part of a properly distributed sequence at order 1. This is equivalent to the statement (see [7, Theorem 1.4.2]) that $\Lambda$ is part (with the multiplicities $m_{k}$ ) of the zero set of an entire function of exponential type and completely regular growth. We denote by $F(\Lambda)$ the collection of all entire functions of exponential type and completely regular growth whose zero sets contain $\Lambda$.

Let $f(z)$ be an entire function of exponential type. Its (upper) indicator is defined to be the function

$$
h_{f}(\lambda)=\limsup _{t \rightarrow \infty} \frac{\ln |f(t \lambda)|}{t}, \quad \lambda \in \mathbb{C} .
$$

If $K$ is the convex compact set coinciding with the conjugate diagram of $f$, then by the Polya theorem (see, e.g., 9, Theorem 5.4]) we have

$$
h_{f}(\lambda)=H_{K}(\lambda), \quad \lambda \in \mathbb{C} .
$$

We denote by $\underline{h}_{f}$ the lower indicator of $f$ (see [17, Chapter 4]):

$$
\underline{h}_{f}=\lim _{\delta \rightarrow 0} \liminf _{t \rightarrow \infty} \frac{1}{\pi \delta^{2}} \int_{B(t \lambda, t|\lambda|)} \frac{\ln |f(z)|}{t} d x d y, \quad z=x+i y .
$$

The definitions show that the lower indicator does not exceed the upper. The function $f$ is of completely regular growth (see [17, Chapter 4]) if and only if

$$
h_{f}(\lambda)=\underline{h}_{f}(\lambda), \quad \lambda \in \mathbb{C} .
$$

Lemma 4.1. Let $\Lambda$ be a proper sequence. Then $\sigma(\Lambda)=0$ and $M_{\Lambda}=0$.

Proof. By assumption, $\Lambda$ is part of the zero set of an entire function of exponential type. Therefore, $N(\Lambda)<\infty$ (see, e.g., [7, Theorem 1.1.2]). This directly shows that $\sigma(\Lambda)=0$. We prove that $M_{\Lambda}=0$. We argue by contradiction.

Suppose that $M_{\Lambda} \geq \tau>0$. Using the definition of $M_{\Lambda}$, we choose a subsequence $\left\{\lambda_{k(p)}\right\}$ of $\left\{\lambda_{k}\right\}$ such that

$$
\liminf _{p \rightarrow \infty} \frac{M_{\Lambda}\left(\lambda_{k(p)}, 1 / p\right)}{\left|\lambda_{k(p)}\right|} \geq \tau .
$$

Another passage to a subsequence with (2.2) taken into account allows us to assume that the sequence $\left\{\lambda_{k(p)} /\left|\lambda_{k(p)}\right|\right\}$ converges to a point $\xi \in \mathbb{S}$. Let $f \in F(\Lambda)$ and $\varepsilon>0$. The properties of the indicators (see, e.g., [18, Chapter 1, §6, Subsection 2]) imply the existence of positive numbers $T$ and $\delta<1$ with

$$
\frac{\ln |f(t \eta)|}{t} \leq h_{f}(\xi)+\varepsilon, \quad \eta \in B(\xi, 3 \delta), \quad t \geq T
$$

Since $f$ is of completely regular growth, we have (see [7, Chapter. I, §4, Subsection 1])

$$
\lim _{t \rightarrow \infty, t \notin E} \frac{\ln |f(t \xi)|}{t}=h_{f}(\xi),
$$


where $E$ is a set of zero relative measure, i.e.,

$$
\lim _{r \rightarrow \infty} \frac{\operatorname{meas}(E \cap[0, r])}{r}=0 .
$$

Outside $E$, we choose an unbounded monotone increasing sequence $\left\{t_{l}\right\}$ positive numbers with

$$
\lim _{l \rightarrow \infty} \frac{t_{l+1}}{t_{l}}=1
$$

This can be done because, by (4.19), for every $\alpha>1$ we have

$$
\lim _{r \rightarrow \infty} \frac{\operatorname{meas}(E \cap[r, \alpha r])}{r}=0
$$

i.e., every interval $\left[r, \alpha_{r}\right]$ with $r$ sufficiently large contains points not belonging to $E$. By (4.3), we can find $l_{0}$ such that

$$
\ln \left|f\left(t_{l} \xi\right)\right| \geq\left(h_{f}(\xi)-\varepsilon\right) t_{l}
$$

and $t_{l} \geq T$ for every $l \geq l_{0}$. Consider the functions

$$
f_{l}(\varsigma)=\frac{f\left(t_{l} \xi+\varsigma\right)}{f\left(t_{l} \xi\right)}, \quad l \geq l_{0}
$$

Formulas (4.2) and (4.6) show that

$$
\ln \left|f_{l}(\varsigma)\right| \leq 2 \varepsilon t_{l}, \quad \varsigma \in B\left(0,3 \delta t_{l}\right), \quad l \geq l_{0} .
$$

Let $n(l)$ be the number of zeros (with multiplicities) of the function $f_{l}(\varsigma)$ in the disk $B\left(0,3 \delta t_{l} / e\right)$. By the theorem on the zeros of an analytic function in a disk (see 9, Theorem 2.1]), we have

$$
\frac{\left(\delta t_{l}\right)^{n(l)}}{\left|\varsigma_{1} \varsigma_{2} \ldots \varsigma_{n(l)}\right|} \leq \exp \left(2 \varepsilon t_{l}\right), \quad l \geq l_{0}
$$

where $\varsigma_{1}, \ldots, \varsigma_{n(l)}$ are the zeros of $f_{l}$ in the disk $B\left(0,3 \delta t_{l} / e\right)$. Since $\left|\varsigma_{j}\right| \leq 3 \delta t_{l} / e$, it follows that

$$
e^{n(l)} \leq \frac{\left(3 \delta t_{l}\right)^{n(l)}}{\left|\varsigma_{1} \varsigma_{2} \ldots \varsigma_{n(l)}\right|} \leq \exp \left(2 \varepsilon t_{l}\right), \quad l \geq l_{0} .
$$

Thus, recalling (2.2) and the definition of $f_{l}(\varsigma)$, we arrive at

$$
M_{\Lambda}\left(t_{l} \xi, \delta\right) \leq 2 \varepsilon t_{l}, \quad l \geq l_{0} .
$$

By (4.5), increasing $l_{0}$ if necessary, we may assume that

$$
t_{l+1} \leq(1+\delta / 4) t_{l} .
$$

We choose an index $p_{0}$ such that

$$
\left|\lambda_{k(p)} /\right| \lambda_{k(p)}|-\xi| \leq \delta / 4, \quad\left|\lambda_{k(p)}\right| \geq t_{l_{0}}
$$

for all $p \geq p_{0}$. Fixing $p \geq p_{0}$, we choose $l(p) \geq l_{0}$ with

$$
t_{l(p)} \leq\left|\lambda_{k(p)}\right| \leq t_{l(p)+1} .
$$

Let $\lambda$ be an arbitrary point of the disk $B\left(\lambda_{k(p)}, \delta\left|\lambda_{k(p)}\right| / 4\right)$. Then

$$
\begin{aligned}
\left|\lambda-t_{l(p)} \xi\right| & \leq\left|\lambda-\lambda_{k(p)}\right|+\left|\lambda_{k(p)}-t_{l(p)} \xi\right| \\
& \leq \delta\left|\lambda_{k(p)}\right| / 4+\left|\lambda_{k(p)}-\right| \lambda_{k(p)}|\xi|+|| \lambda_{k(p)}\left|\xi+t_{l(p)} \xi\right| \\
& \leq \delta\left|\lambda_{k(p)}\right| / 2+\left|\lambda_{k(p)}\right|-t_{l(p)} \leq \delta t_{l(p)} / 2+t_{l(p)}-t_{l(p)} \\
& \leq \delta(1+\delta / 4) t_{l(p)} / 2+\delta t_{l(p)} / 4<\delta t_{l(p)} .
\end{aligned}
$$


Consequently, the disk $B\left(\lambda_{k(p)}, \delta\left|\lambda_{k(p)}\right| / 4\right)$ is included in $B\left(t_{l(p)} \xi, \delta t_{l(p)}\right)$. Therefore, by (2.2) and (4.7), we have

$$
M_{\Lambda}\left(\lambda_{k(p)}, \delta\left|\lambda_{k(p)}\right| / 4\right) \leq M_{\Lambda}\left(t_{l(p)} \xi, \delta\right) \leq 2 \varepsilon t_{l(p)} \leq 2 \varepsilon\left|\lambda_{k(p)}\right| .
$$

Together with (2.2), this implies that

$$
M_{\Lambda}\left(\lambda_{k(p)}, 1 / p\right) \leq 2 \varepsilon\left|\lambda_{k(p)}\right|
$$

for $p$ sufficiently large. This contradicts (4.1) because $\varepsilon>0$ can be chosen arbitrarily small. The lemma is proved.

The next statement is an immediate consequence of Theorem 5.2 in [14.

Lemma 4.2. Let $\Lambda$ be a proper sequence, and let $S_{\Lambda}=0$. Next, let $f \in F(\Lambda)$, let $K$ be the conjugate diagram of $f$, and let $w \in \mathbb{C}$ and $r>0$. Suppose that $g$ can be approximated, uniformly on compact sets in $D=K+B(w, r)$, by linear combinations of elements of the system $\mathcal{E}=\left\{z^{n} \exp \left(\lambda_{k} z\right)\right\}_{\substack{\infty, m_{k}-1 \\ k=1, n=0}}$. Then, in this domain, $g$ is uniquely representable by the series (1.1).

Proof. We show that the assumptions of Theorem 5.2 in [14] are satisfied. Let $W$ denote the closure of the linear hull of $\mathcal{E}$ in the space $H(D)$ of functions analytic in $D$, with the topology of uniform convergence on compact sets. Then $W$ is a closed subspace of $H(D)$ invariant under differentiation, admitting spectral synthesis, and having $\Lambda=\left\{\lambda_{k}, m_{k}\right\}_{k=1}^{\infty}$ as the spectrum.

We put $f_{1}(\lambda)=f(\lambda) \exp (\lambda w)$. Since $K$ is the conjugate diagram of $f$, by the definition of $D$ we obtain

$$
h_{f_{1}}(\lambda)=\operatorname{Re}(\lambda w)+h_{f}(\lambda)=\operatorname{Re}(\lambda w)+H_{K}(\lambda)<H_{D}(\lambda), \quad \lambda \in \mathbb{C}, \quad \lambda \neq 0 .
$$

By the Polya theorem (see, e.g., [18]) this inequality suffices for the existence of a continuous linear functional $\mu$ on $H(D)$ whose Laplace transform $\widehat{\mu}(\lambda)=(\mu, \exp (\lambda z))$ coincides with $f_{1}(\lambda)$. It is easily seen that the value of this functional at the function $z^{n} \exp \left(\lambda_{k} z\right)$ is equal to $f_{1}^{(n)}\left(\lambda_{k}\right)$. By the definition of $F(\Lambda)$, this means that the nonzero functional $\mu$ vanishes at all functions of the system $\mathcal{E}$ and, consequently, on $W$. Therefore, $W$ is a proper subspace of $H(D)$.

Let $f_{2}(\lambda)$ be an entire function of exponential type and completely regular growth whose indicator $h_{f_{2}}(\lambda)$ is equal to the support function of the disk $B(w, r)$, i.e.,

$$
h_{f_{2}}(\lambda)=H_{B(w, r)}(\lambda)=\operatorname{Re}(\lambda w)+r|\lambda|, \quad \lambda \in \mathbb{C} .
$$

The existence of such a function was established, e.g., in a theorem in [7]. We put $\varphi(\lambda)=f_{2}(\lambda) f(\lambda), \lambda \in \mathbb{C}$. Since $\varphi$ is a product of two functions of completely regular growth, $\varphi$ itself is of completely regular growth (this follows directly from the definition), i.e.,

$$
h_{\varphi}(\lambda)=\underline{h}_{\varphi}(\lambda), \quad \lambda \in \mathbb{C} .
$$

Moreover, by the theorem on addition of indicators (see, e.g., [7, Theorem 1.4.3]), we have

$$
h_{\varphi}(\lambda)=h_{f_{2}}(\lambda)+h_{f}(\lambda)=H_{B(w, r)}(\lambda)+H_{K}(\lambda)=H_{D}(\lambda), \quad \lambda \in \mathbb{C} .
$$

Note that $S_{\Lambda}=0$ by assumption, and $m(\Lambda) \leq M_{\Lambda}=0$ by Lemma 4.1. Thus, the hypotheses of Theorem 5.2 in [14] are fulfilled. By that theorem, every function in $W$ (in particular, $g$ ) is representable by a series of the form (1.1). Also in [14], it was proved that such a representation is unique. The lemma is proved. 
For an open set $D$ and a compact set $K$, we denote by $\Omega(D, K)$ the collection of all $z$ such that $K+z$ (a translate of $K$ ) is included in $D$. If $D$ is a convex domain, it is easily seen that $\Omega(D, K)$ is also a convex domain (which may be empty). Another way to define this domain is to put

$$
\Omega(D, K)=\left\{z \in \mathbb{C}: \operatorname{Re}(z \lambda)<H_{D}(\lambda)-H_{K}(\lambda), \lambda \in \mathbb{S}\right\} .
$$

Theorem 4.1. Let $\Lambda$ be a proper sequence, let $f \in F(\Lambda)$, and let $K$ be the conjugate diagram of $f$. The following statements are equivalent.

1) For every sequence $d \in \mathfrak{A}(\Lambda)$ with the property that the set $\Omega(D(\Lambda, d), K)$ is nonempty and is different from the plane, and for every $w \in \partial \Omega(D(\Lambda, d), K)$, the function $g_{d}(z)$ has at least one singular point in $(w+K) \cap \partial D(\Lambda, d)$.

2) $S_{\Lambda}=0$.

Proof. 1) $\Longrightarrow 2$ ). Suppose that $S_{\Lambda}<0$. Let $D$ be a bounded convex domain that includes $K$. Lemma 4.1 shows that $m(\Lambda) \leq M_{\Lambda}=0$. By Theorem 3.1 and the remark to its statement, there exists a sequence $d \in \mathfrak{A}(\Lambda)$ such that the set $D(\Lambda, d)$ is not the entire plane and the function $g_{d}(z)$ has no singular points on the boundary $\partial D(\Lambda, d)$. Since the set $\Omega(D(\Lambda, d), K)$ is nonempty in this case (it includes $K$ ), this contradicts statement 1 ). When defining $S_{\Lambda}$, we observed that $S_{\Lambda}$ is always nonpositive. Thus, $S_{\Lambda}=0$.

$2) \Longrightarrow 1)$. Suppose a sequence $d \in \mathfrak{A}(\Lambda)$ has the property that $\Omega(D(\Lambda, d), K)$ is nonempty. Note that $\sigma(\Lambda)=m(\Lambda)=0$ by Lemma 4.1. Consequently, by the CauchyHadamard theorem for series of exponential monomials, the set $D(\Lambda, d)$ and, with it, the set $\Omega(D(\Lambda, d), K)$ are convex domains. We fix a point $w_{0} \in \partial \Omega(D(\Lambda, d), K)$ and suppose that $g_{d}(z)$ has no singular points on the compact set $\left(w_{0}+K\right) \cap \partial D(\Lambda, d)$. In other words, for every $w \in\left(w_{0}+K\right) \cap \partial D(\Lambda, d)$ the function $g_{d}$ admits analytic continuation to some disk $B(w, r(w))$. If the disks $B\left(w_{1}, r\left(w_{1}\right)\right)$ and $B\left(w_{2}, r\left(w_{2}\right)\right)$ intersect, then, since $D(\Lambda, d)$ is convex, the intersection $B\left(w_{1}, r\left(w_{1}\right)\right) \cap B\left(w_{2}, r\left(w_{2}\right)\right) \cap D(\Lambda, d)$ is also nonempty. By the uniqueness theorem, the extensions of $g_{d}(z)$ to the disks $B\left(w_{1}, r\left(w_{1}\right)\right)$ and $B\left(w_{2}, r\left(w_{2}\right)\right)$ coincide on the intersection of these disks. Thus, $g_{d}(z)$ admits analytic continuation to the domain $D(\Lambda, d) \cup U$, where $U$ is a neighborhood of the compact set $\left(w_{0}+K\right) \cap \partial D(\Lambda, d)$.

We choose a positive number $r$ with $K+B\left(w_{0}, r\right) \subseteq D(\Lambda, d) \cup U$. The function $g_{d}(z)$ can be approximated uniformly on compact sets by linear combinations of elements of the system $\mathfrak{E}$ in some neighborhood of a translate of $K$ (for instance, in $D(\Lambda, d)$ ). By a result in [19], this approximation extends to the domain $K+B\left(w_{0}, r\right)$. By Lemma 4.2, it follows that $g_{d}(z)$ is representable by a series of the form (1.1) in this domain, maybe with coefficients different from $d=\left\{d_{k, n}\right\}$. We show that, in fact, these coefficients coincide with $d=\left\{d_{k, n}\right\}$. Let $\widetilde{w}$ be an arbitrary common point of the disk $B\left(w_{0}, r\right)$ and the domain $\Omega(D(\Lambda, d), K)$. We choose $\widetilde{r}>0$ such that the disk $B(\widetilde{w}, \widetilde{r})$ lies in the intersection $B\left(w_{0}, r\right) \cap \Omega(D(\Lambda, d), K)$. Then the domain $K+B(\widetilde{w}, \widetilde{r})$ is included both in $K+B\left(w_{0}, r\right)$ and in $D(\Lambda, d)$. Hence, in $K+B(\widetilde{w}, \widetilde{r})$ there are two representations of $g_{d}(z)$ by a series (1.1). However, by Lemma 4.2 such a representation is unique, i.e., the coefficients of the two representations coincide. We have proved that, in the domain $D(\Lambda, d) \cup\left(K+B\left(w_{0}, r\right)\right)$ the function $g_{d}(z)$ expands in the series (1.1) with the coefficients $d=\left\{d_{k, n}\right\}$. This contradicts the definition of $D(\Lambda)$, because the domain $D(\Lambda, d) \cup\left(K+B\left(w_{0}, r\right)\right)$ is wider than $D(\Lambda, d)$. Consequently, our supposition is not true, i.e., $g_{d}(z)$ has at least one singular point in $\left(w_{0}+K\right) \cap \partial D(\Lambda, d)$. The theorem is proved.

Remark. Theorem 3.1 implies that if $S_{\Lambda} \neq 0$, then there exists a series of the form (1.1) with an arbitrarily large domain of convergence whose sum has no singular points on the boundary of this domain. But if $S_{\Lambda}=0$ and the sequence $\Lambda$ is proper, then, by 
Theorem 4.1, the sum of every series of the form (1.1) with sufficiently large domain of convergence has some singular points on its boundary. However, if this domain is smaller than necessary, then the sum of the series may fail to have any singular points, even though $\Lambda$ may be proper and $S_{\Lambda}$ may be equal to 0 . We give an example (see [9]). Put

$$
L(\lambda)=\frac{\sin \lambda \sin (i \lambda)}{\lambda^{2}} .
$$

The function $L(\lambda)$ is entire, of exponential type, and of completely regular growth, because it is the product of two functions $\sin (\pi \lambda) / \lambda$ and $\sin (i \pi \lambda) / \lambda$ each being of completely regular growth. By the theorem on addition of indicators, the conjugate diagram $K$ of $L$ is the sum of the conjugate diagrams of these two functions, which are the intervals $[-i, i]$ on the imaginary axis and $[-1,1]$ on the real axis, respectively. Therefore, $K$ is the square with vertices at the points $1+i, i-1,1-i,-1-i$. The function $L(\lambda)$ has simple zeros at the points $\pi n$ and $i \pi$. Let $\Lambda=\left\{\lambda_{k}\right\}_{k=1}^{\infty}$ be the sequence of its zeros, and let $d=\left\{1 / L^{\prime}\left(\lambda_{k}\right)\right\}_{k=1}^{\infty}$. Then $\Lambda$ is a proper sequence. As in the examples after the definition of $S_{\Lambda}$, it can be shown that now this quantity is zero. Consider the series

$$
\sum_{k=1}^{\infty} \frac{\exp \left(\lambda_{k} z\right)}{L^{\prime}\left(\lambda_{k}\right)}
$$

In the book [9] it was proved that its sum is identically zero (therefore, has no singular points), and its domain of convergence is the interior of $K$ (the domain of convergence can also easily be calculated with the help of the Cauchy-Hadamard theorem). In a sense, this case is ultimate. Here $K$ is not included in $D(\Lambda, d)$ but coincides with its closure.

Now we show that all results concerting singular points of the sums of exponential series and their particular cases (Dirichlet and Taylor series) that were mentioned in the Introduction are consequences of Theorem 4.1. At the same time, we do not touch upon the results of Lunts [11, 12] and Ostrowski [10] for the reasons explained in the Introduction.

We begin with the results pertaining to the Dirichlet series

$$
g(z)=\sum_{k=1}^{\infty} d_{k} \exp \left(-\lambda_{k} z\right),
$$

where $\Lambda=\left\{\lambda_{k}\right\}_{k=1}^{\infty}$ is an unbounded monotone increasing sequence of positive numbers. We remind the reader (see [7, 9]) that this series converges in a certain half-plane $\operatorname{Re} z>c$, the number $c$ is called the abscissa of convergence and is calculated by the CauchyHadamard formula. The line $\operatorname{Re} z=c$ is called the line of convergence. In the sequel, we assume that the domain of convergence of the series (4.8) is nonempty, i.e., $c>-\infty$.

Corollary 4.1. Suppose the sequence $\Lambda=\left\{\lambda_{k}\right\}$ possesses a density, i.e.,

$$
\tau=N(\Lambda)=\lim _{k \rightarrow \infty} \frac{k}{\lambda_{k}}
$$

Then the following statements are equivalent.

1) Every function $g(z)$ of the form (4.8) is either entire, or has a singular point on every interval of length $2 \pi \tau$ that lies on the convergence line.

2) $S_{\Lambda}=0$.

Proof. Put

$$
L(\lambda)=\prod_{k=1}^{\infty}\left(1-\frac{\lambda^{2}}{\lambda_{k}^{2}}\right) .
$$


By Theorem 1.2.9 in the book [7, $L(\lambda)$ is an entire function of exponential type and completely regular growth, and its conjugate diagram $K$ coincides with the interval $[-i \pi \tau, i \pi \tau]$ on the imaginary axis. Thus, $\Lambda$ is a proper sequence. In our case, the domain $\Omega(D(\Lambda, d), K)$ coincides with the half-plane of convergence for the series (4.8). Therefore, for every $w \in \partial \Omega(D(\Lambda, d), K)$, the set $(w+K) \cap \partial D(\Lambda, d)$ is an interval of length $2 \pi \tau$ on the convergence line. Moreover, any such interval can be obtained in this way. To complete the proof, it remains to apply Theorem 4.1.

Let

$$
\gamma(\Lambda)=\limsup _{k \rightarrow \infty} \frac{1}{\lambda_{k}} \ln \left|\frac{1}{L^{\prime}\left(\lambda_{k}\right)}\right|,
$$

where $L(\Lambda)$ is given by (4.9). The quantity $\gamma(\Lambda)$ is called the Bernstein-Leont'ev condensation index.

Corollary 4.2 (Bernstein theorem). Suppose that a sequence $\lambda$ has density $\tau$, and $\gamma(\Lambda)=0$. Then every function $g(z)$ of the form (4.8) is either entire, or has at least one singular point on every interval of length $2 \pi \tau$ on the convergence line.

Proof. By Corollary 4.1, it suffices to prove that $S_{\Lambda}=0$ whenever $\gamma(\Lambda)=0$. So, let $\gamma(\Lambda)=0$. Then for every $\varepsilon>0$ there exists $k_{0}$ with

$$
\left|L^{\prime}\left(\lambda_{k}\right)\right| \geq \exp \left(-\varepsilon \lambda_{k}\right), \quad k \geq k_{0} .
$$

By the properties of indicators (see, e.g., [18]), there exist $\alpha, T>0$ such that

$$
|L(\lambda)| \leq \exp \left(\left(h_{L}(1)+\varepsilon\right)|\lambda|\right)=\exp \left(\left(H_{K}(1)+\varepsilon\right)|\lambda|\right), \quad \lambda /|\lambda| \in B(1, \alpha), \quad|\lambda| \geq T,
$$

where $K$ is the conjugate diagram for $L(\lambda)$, coinciding with the interval $[-i \pi \tau, i \pi \tau]$ on the imaginary axis. Since $H_{K}(1)=0$, it follows that

$$
|L(\lambda)| \leq \exp (\varepsilon|\lambda|), \quad \lambda /|\lambda| \in B(1,4 \alpha), \quad|\lambda| \geq T .
$$

Let $L(\lambda)=\left(\lambda-\lambda_{k}\right) L_{k}(\lambda)$. Then $L^{\prime}\left(\lambda_{k}\right)=L_{k}\left(\lambda_{k}\right)$. The last estimate implies the inequality

$$
\left|L_{k}\left(\lambda_{k}\right)\right|=\left|\frac{L(\lambda)}{\lambda-\lambda_{k}}\right| \leq 4 \alpha \lambda_{k} \exp \left(\varepsilon(1+4 \alpha) \lambda_{k}\right), \quad \lambda \in S\left(\lambda_{k}, 4 \alpha \lambda_{k}\right), \quad \lambda_{k} \geq T .
$$

Since

$$
\left|q_{\Lambda}^{k}(\lambda, \alpha)\right| \geq 1
$$

on the circle $S\left(\lambda_{k}, 4 \alpha \lambda_{k}\right)$, we see that

$$
\left|\frac{L_{k}(\lambda)}{q_{\Lambda}^{k}(\lambda, \alpha)}\right| \leq 4 \alpha \lambda_{k} \exp \left(\varepsilon(1+4 \alpha) \lambda_{k}\right), \quad \lambda \in S\left(\lambda_{k}, 4 \alpha \lambda_{k}\right), \quad \lambda_{k} \geq T .
$$

By the maximum principle, this inequality extends to the interior of the disk. In particular,

$$
\left|\frac{L_{k}(\lambda)}{q_{\Lambda}^{k}(\lambda, \alpha)}\right| \leq 4 \alpha \lambda_{k} \exp \left(\varepsilon(1+4 \alpha) \lambda_{k}\right), \quad \lambda_{k} \geq T .
$$

Taking (4.10) into account, we obtain

$$
\begin{aligned}
\left|q_{\Lambda}^{k}(\lambda, \alpha)\right| & \geq\left|L_{k}\left(\lambda_{k}\right)\right| \exp \left(-\varepsilon(1+4 \alpha) \lambda_{k}-\ln \left(4 \alpha \lambda_{k}\right)\right) \\
& =\left|L^{\prime}\left(\lambda_{k}\right)\right| \exp \left(-\varepsilon(1+4 \alpha) \lambda_{k}-\ln \left(4 \alpha \lambda_{k}\right)\right) \\
& \geq \exp \left(-\varepsilon(2+4 \alpha) \lambda_{k}-\ln \left(4 \alpha \lambda_{k}\right)\right)
\end{aligned}
$$

for all indices $k$ with $\lambda_{k} \geq T$ and $k \geq k_{0}$. Then the definition of $S_{\Lambda}$ shows that $S_{\Lambda} \geq-\varepsilon$. Since $\varepsilon>0$ is arbitrary, we see that $S_{\Lambda} \geq 0$. The reverse inequality $S_{\Lambda} \leq 0$ is always true. Thus, $S_{\Lambda}=0$ and the corollary is proved. 
Corollary 4.3 (Polya theorem). Suppose a sequence $\Lambda=\left\{\lambda_{k}\right\}$ has density $\tau$ and $\lambda_{k+1}-$ $\lambda_{k} \geq h>0, k=1,2, \ldots$. Then every function $g(z)$ of the form (4.8) either is entire or has at least one singular point on an arbitrary interval of length $2 \pi \tau$ on the convergence line.

Proof. As above, it suffices to show that $S_{\Lambda}=0$. But this was done in an example after the definition of $S_{\Lambda}$.

It was mentioned in the Introduction that the other results quoted there are also consequences of the facts established in this paper. In particular, this concerns the theorems by Hadamard, Fabry, Carlson, and Landau. All of them deal with positive sequences of zero density. Now we consider the case of an arbitrary sequence $\Lambda=$ $\left\{\lambda_{k}, m_{k}\right\}_{k=1}^{\infty}$ with zero density.

Corollary 4.4. Suppose that a sequence $\Lambda=\left\{\lambda_{k}, m_{k}\right\}_{k=1}^{\infty}$ has zero density. Then the following statements are equivalent.

1) For every sequence $d \in \mathfrak{A}(\Lambda)$, the function $g_{d}(z)$ either is entire or has singularities at all boundary points of $D(\Lambda, d)$. In particular, the domain of existence of $g_{d}(z)$ is convex.

2) We have $S_{\Lambda}=0$.

Proof. Put

$$
L(\lambda)=\prod_{k=1}^{\infty}\left(1-\frac{\lambda^{2}}{\lambda_{k}^{2}}\right)^{m_{k}} .
$$

Since the sequence $\Lambda$ has zero density, $L(\lambda)$ is an entire function of minimal type (see, e.g., [7) and, consequently, of completely regular growth. Its conjugate diagram is the singleton consisting of the origin. Thus, $\Lambda$ is a proper sequence. As in Theorem 4.1, for every sequence $d \in \mathfrak{A}(\Lambda)$ the set $D(\Lambda, d)$ is a convex domain. Since $K$ is a singleton, the domain $\Omega(D(\Lambda, d), K)$ is nonempty and coincides with $D(\Lambda, d)$. Moreover, every $z \in \partial D(\Lambda, d)$ (which is also a boundary point for $\Omega(D(\Lambda, d), K)$ ) is a unique element of the intersection $(z+K) \cap \partial(D(\Lambda, d)$. It remains to apply Theorem 4.1.

We state a particular case of the above corollary.

Corollary 4.5 (Leont'ev's theorem). Suppose that a sequence $\Lambda=\left\{\lambda_{k}, m_{k}\right\}_{k=1}^{\infty}$ has zero density and $\gamma(\Lambda)=0$. Then for every sequence $d \in \mathfrak{A}(\Lambda)$ the function $g_{d}(z)$ either is entire, or has singularities at all boundary points of $D(\Lambda, d)$. In particular, the domain of existence of $g_{d}(z)$ is convex.

Proof. It suffices to show that $S_{\Lambda}=0$. This is done much as in Corollary 4.2.

Theorem 4.1 implies that the case of zero density is not the only case in which all points $z \in D(\Lambda, d)$ are singular for $g_{d}$. Indeed, the same feature occurs if every boundary point of $D(\Lambda, d)$ coincides with a set of the form $(w+K) \cap \partial D(\Lambda, d)$, where $w \in \partial \Omega(D(\Lambda, d), K)$. We present some conditions guaranteeing this. In this connection, we remind the reader that (see [16]) a convex domain has smooth boundary if and only if only one support line passes through each boundary point. Next, a compact convex set is strictly convex if and only if every support line meets it at a unique point. In particular, a singleton is strictly convex, but an interval is not because two of its support lines include the entire interval.

Lemma 4.3. Let $\Omega$ be a convex domain with smooth boundary, and let $K$ be a strictly convex compact set. Then every boundary point of the domain $D=\Omega+K$ coincides with the intersection $(w+K) \cap \partial D$, where $w$ is a boundary point of $\Omega$. 
Proof. Let $z \in \partial D$. Since $D$ is the sum of $\Omega$ and $K$, there exist $w \in \partial \Omega$ and $v \in K$ with $z=w+v$. Thus, $z \in(w+K) \cap \partial D$. If $z$ is a unique point of this intersection, the proof is finished. Suppose there exists $z_{1}$ different from $z$ and belonging to $(w+K) \cap \partial D$. Then $z_{1}=w+v_{1}$, where $v_{1} \in K$. At least one support line for $D$ passes through each of the points $z$ and $z_{1}$. We denote these lines by $l=\left\{u \in \mathbb{C}: \operatorname{Re}(u \xi)=H_{D}(\xi)\right\}$ and $l_{1}=\left\{u \in \mathbb{C}: \operatorname{Re}\left(u \xi_{1}\right)=H_{D}\left(\xi_{1}\right)\right\}$, where $\xi, \xi_{1} \in \mathbb{S}$. We claim that

$$
\operatorname{Re}(w \xi)=H_{\Omega}(\xi), \operatorname{Re}\left(w \xi_{1}\right)=H_{\Omega}\left(\xi_{1}\right), \operatorname{Re}(v \xi)=H_{K}(\xi), \operatorname{Re}\left(v_{1} \xi_{1}\right)=H_{K}\left(\xi_{1}\right) .
$$

Indeed, suppose that, for instance, $\operatorname{Re}(w \xi) \neq H_{\Omega}(\xi)$. Since $w$ belongs to the closure of $\Omega$, we have $\operatorname{Re}(w \xi) \leq H_{\Omega}(\xi)$ by the definition of the support function. Consequently, $\operatorname{Re}(w \xi)<H_{\Omega}(\xi)$. Then, by the same definition, there exists $\widetilde{w} \in \Omega$ such that $\operatorname{Re}(w \xi)<$ $\operatorname{Re}(\widetilde{w} \xi)$. Since $z \in l$, we obtain

$$
\begin{aligned}
\operatorname{Re}((\widetilde{w}+v) \xi) & =\operatorname{Re}(\tilde{w} \xi)+\operatorname{Re}(v \xi)>\operatorname{Re}(w \xi)+\operatorname{Re}(v \xi) \\
& =\operatorname{Re}((w+v) \xi)=\operatorname{Re}(z \xi)=H_{D}(\xi) .
\end{aligned}
$$

On the other hand, $\widetilde{w}+v \in \Omega+K=D$, and the definition of the support function for $D$ implies the inequality $\operatorname{Re}((\widetilde{w}+v) \xi) \leq H_{D}(\xi)$. This contradiction proves the above claim. The first two identities claimed and proved mean that $w \in \partial \Omega$ belongs to two support lines for $\Omega$. But $\Omega$ is smooth by assumption, so $\xi=\xi_{1}$. Then the other two identities mean that different points $v, v_{1} \in K$ belong to one and the same support line for $K$. This is impossible because $K$ is a strictly convex compact set by assumption.

Thus, the supposition that $(w+K) \cap \partial D$ contains a point distinct from $z$ is not true, which proves the lemma.

The following statement is an immediate consequence of Lemma 4.3 and Theorem 4.1.

Corollary 4.6. Let $\Lambda$ be a proper sequence, and let $f \in F(\Lambda)$. Denote by $K$ the conjugate diagram for $f$ and take $d \in \mathfrak{A}(\Lambda)$. Suppose that $S_{\Lambda}=0, K$ is a strictly convex compact set, the set $\Omega(D(\Lambda, d), K)$ is nonempty and differs from the entire plane, the boundary of the domain $D(\Lambda, d)$ is smooth, and $D(\Lambda, d)=\Omega(D(\Lambda, d), K)+K$. Then all boundary points of $D(\Lambda, d)$ are singular for $g_{d}(z)$. In particular, the domain of existence of this function is convex.

Remark. We give an example to show how Corollary 4.6 applies. Let $K$ be an arbitrary strictly convex compact set different from a singleton. Then $K$ is the conjugate diagram for some entire function $f$ of exponential type, of completely regular growth, and with simple zeros $\Lambda=\left\{\lambda_{k}\right\}$, see [9], 7, Theorem 1.3.2]. Moreover, the sequence $\Lambda$ satisfies the condition lim $\ln \left|f^{\prime}\left(\lambda_{k}\right)\right| /\left|\lambda_{k}\right|=0$ (as in Corollary 4.2, this condition implies $S_{\Lambda}=0$ ). Specifically, $f$ is the canonical function for a certain properly distributed (and even regular) set $\Lambda=\left\{\lambda_{k}\right\}$. This set is constructed in such a way that $\Theta(\Lambda)$ coincides with the circle $\mathbb{S}$. Let $\Omega$ be an arbitrary convex domain with smooth boundary. Putting $D=\Omega+K$, we define the coefficients $d=\left\{d_{k}\right\}$ as follows:

$$
d_{k}=c_{k} \exp \left(-H_{K_{p(k)}}\left(\lambda_{k}\right)\right), \quad k=1,2, \ldots,
$$

where $\left\{K_{p}\right\}$ is a sequence of compact convex sets exhausting $D, p(k) \rightarrow \infty$ as $k \rightarrow \infty$, and $\left\{c_{k}\right\}$ is an arbitrary sequence of complex numbers satisfying the condition $\ln \left|c_{k}\right| /\left|\lambda_{k}\right| \rightarrow 0$ as $k \rightarrow \infty$. Let

$$
g(z)=\sum_{k=1}^{\infty} d_{k} \exp \left(\lambda_{k} z\right) .
$$

Since $\Theta(\Lambda)=\mathbb{S}$, as in Theorem 3.1 we conclude, by using the Cauchy-Hadamard theorem for series of exponentials, that the convergence domain $D(\Lambda, d)$ for this series coincides 
with $D$. Then $\Omega(D(\Lambda, d), K)=\Omega$. Thus, the assumptions of Corollary 4.6 are satisfied. Consequently, the domain of existence for $g(z)$ coincides with the convex domain $D(\Lambda, d)$.

\section{§5. THE CASE OF ZERO DENSITY}

In this section we shall show that the condition $N(\Lambda)=0$ is necessary for item 1 ) in Corollary 4.4. In other words, we shall obtain a criterion for equality between the domain of convergence for the series (1.1) and the domain of existence of its every sum. To do this, we need two auxiliary statements.

Lemma 5.1. Suppose that a sequence $\Lambda$ satisfies $m(\Lambda) \neq 0$. Then there exists a sequence $d \in \mathfrak{A}(\Lambda)$ of coefficients such that all boundary points of $D(\Lambda, d)$ are singular for $g_{d}(z)$.

Proof. Let $m(\Lambda) \geq 2 \rho>0$. We may assume that $\rho<1 / 2 e^{2}$. By the definition of $m(\Lambda)$, there exists a subsequence $\left\{\lambda_{k(p)}\right\}$ satisfying

$$
m(k(p)) \geq \rho\left|\lambda_{k(p)}\right|, \quad p=1,2, \ldots
$$

Passing to a subsequence once again, we may assume that the sequence $\left\{\lambda_{k(p)} /\left|\lambda_{k(p)}\right|\right\}$ converges to some point $\varsigma$ on the circle $\mathbb{S}$ and that $\left|\lambda_{k(p)}\right| \geq p, p=1,2, \ldots$ Let $n(p)$ be the integral part of $\rho\left|\lambda_{k(p)}\right|, p=1,2, \ldots$ Consider the function

$$
g(z)=\sum_{p=1}^{\infty} c_{p}(z-\bar{\varsigma})^{n(p)} \exp \left(\lambda_{k(p)} z\right),
$$

where $c_{p}=\exp \left(-(1-1 / p)\left|\lambda_{k(p)}\right|\right)$. We show that the series converges in a neighborhood of $\bar{\varsigma}$. We have

$$
\begin{array}{r}
\left|c_{p}(z-\bar{\zeta})^{n(p)} \exp \left(\lambda_{k(p)} z\right)\right| \leq \exp \left(-(1-1 / p)\left|\lambda_{k(p)}\right|+n(p) \ln r+\operatorname{Re}\left(\lambda_{k(p)} \bar{\varsigma}\right)+\left|\lambda_{k(p)}\right| r\right) \\
\leq \exp \left(-(1-1 / p)\left|\lambda_{k(p)}\right|+\left(\rho\left|\lambda_{k(p)}\right|-1\right) \ln r+\operatorname{Re}\left(\lambda_{k(p)} \bar{\varsigma}\right)+\left|\lambda_{k(p)}\right| r\right)
\end{array}
$$

for all $z \in B(\bar{\varsigma}, r), r \in(0,1)$. By the choice of the subsequence, we have $\lambda_{k(p)} /\left|\lambda_{k(p)}\right|=$ $\varsigma+\xi_{p}$, where $\xi_{p} \rightarrow 0$ as $p \rightarrow \infty$. Then, by the above,

$$
\begin{aligned}
\mid c_{p}(z & -\bar{\varsigma})^{n(p)} \exp \left(\lambda_{k(p)} z\right) \mid \\
& \leq \exp \left(-(1-1 / p)\left|\lambda_{k(p)}\right|+\left(\rho\left|\lambda_{k(p)}\right|-1\right) \ln r+\left|\lambda_{k(p)}\right| \operatorname{Re}\left(\bar{\varsigma} \lambda_{k(p)} /\left|\lambda_{k(p)}\right|\right)+\left|\lambda_{k(p)}\right| r\right) \\
& \leq \exp \left(-(1-1 / p)\left|\lambda_{k(p)}\right|+\left(\rho\left|\lambda_{k(p)}\right|-1\right) \ln r+\left|\lambda_{k(p)}\right|\left(1+\left|\xi_{p}\right|\right)+\left|\lambda_{k(p)}\right| r\right) \\
& =\exp \left(\left(1 / p+\rho \ln r+\left|\xi_{p}\right|+r\right)\left|\lambda_{k(p)}\right|-\ln r\right) \leq r_{0}^{-1} \exp \left(-\alpha\left|\lambda_{k(p)}\right|\right),
\end{aligned}
$$

where $\alpha>0$ if $\rho \ln r_{0}<0$ and $p \geq p_{0}$. Since $\rho<1 / 2 e^{2}$, we may assume that $r_{0}=2 \rho$. Since $\left|\lambda_{k(p)}\right| \geq p$ by construction, we have

$$
\left|c_{p}(z-\bar{\varsigma})^{n(p)} \exp \left(\lambda_{k(p)} z\right)\right| \leq r_{0}^{-1} \exp (-\alpha p), \quad p \geq p_{0} .
$$

This means that the series (5.1) converges uniformly in the disk $B\left(\bar{\varsigma}, r_{0}\right)$. Therefore, its sum $g(z)$ is a function analytic in this disk. Consider the series

$$
\sum_{p=1, n=0}^{\infty, n(p)} c_{p} \tilde{d}_{p, n} z^{n} \exp \left(\lambda_{k(p)} z\right),
$$

obtained from (5.1) by expanding the powers of $z-\bar{\varsigma}$. We estimate the coefficients $\widetilde{d}_{p, n}$. We have

$$
(z-\bar{\varsigma})^{n(p)}=\sum_{n=0}^{n(p)} \widetilde{d}_{p, n} z^{n} .
$$


By the Cauchy inequality,

$$
\left|\widetilde{d}_{p, n}\right| \leq \max _{|z|=1}\left|(z-\bar{\varsigma})^{n(p)}\right| \leq \max _{z \in B(\bar{\varsigma}, 2)}\left|(z-\bar{\varsigma})^{n(p)}\right| \leq 2^{n(p)} \leq \exp \left(\rho\left|\lambda_{k(p)}\right|\right) .
$$

Let $z$ be an arbitrary point in the intersection of the disk $B(0,1)$ and the half-plane $\operatorname{Re}(z \varsigma)<1-\rho-\beta, \beta>0$. Then we have

$$
\begin{aligned}
& \sum_{p=1, n=0}^{\infty, n(p)}\left|c_{p} \tilde{d}_{p, n} z^{n} \exp \left(\lambda_{k(p)} z\right)\right| \\
& \quad \leq \sum_{p=1}^{\infty}(n(p)+1) \exp \left((-(1-1 / p)+\rho)\left|\lambda_{k(p)}\right|+\operatorname{Re}\left(\lambda_{k(p)} z\right)\right) \\
& \quad \leq \sum_{p=1}^{\infty}(n(p)+1) \exp \left((-(1-1 / p)+\rho)\left|\lambda_{k(p)}\right|+\left|\lambda_{k(p)}\right| \operatorname{Re}\left(z \lambda_{k(p)} /\left|\lambda_{k(p)}\right|\right)\right. \\
& \quad=\sum_{p=1}^{\infty}(n(p)+1) \exp \left((-(1-1 / p)+\rho)\left|\lambda_{k(p)}\right|+\left|\lambda_{k(p)}\right| \operatorname{Re}\left(z\left(\varsigma+\xi_{p}\right)\right)\right. \\
& \leq \sum_{p=1}^{\infty}(n(p)+1) \exp \left((-(1-1 / p)+\rho)\left|\lambda_{k(p)}\right|+(1-\rho-\beta)\left|\lambda_{k(p)}\right|+\left|\xi_{p}\right|\left|\lambda_{k(p)}\right|\right) \\
& \leq \sum_{p=1}^{\infty}\left(\rho\left|\lambda_{k(p)}\right|+1\right) \exp \left(\left|\lambda_{k(p)}\right| / p-\beta\left|\lambda_{k(p)}\right|+\left|\xi_{p}\right|\left|\lambda_{k(p)}\right|\right)<\infty .
\end{aligned}
$$

The last estimate is a consequence of the relations $\left|\xi_{p}\right| \rightarrow 0$ and $\left|\lambda_{k(p)}\right| \geq p$. Consequently, the series (5.2) converges on the set

$$
B(0,1) \cap\{z: \operatorname{Re}(z \varsigma)<1-\rho-\beta\} .
$$

If $\beta<\rho$, this set includes the nonempty intersection

$$
B(0,1-\rho-\beta) \cap B(\bar{\varsigma}, 2 \rho) .
$$

On this intersection, the sum of the series (5.2) coincides with $g(z)$ because the partial sums of the series (5.1) are at the same time partial sums of the series (5.2).

We put $d_{k, n}=c_{p} \tilde{d}_{p, n}$ if $k=k(p), n=0,1, \ldots, n(p)$, and $d_{k, n}=0$ otherwise. We have arrived at a sequence (namely, $d=\left\{d_{k, n}\right\}$ ) such that $d \in \mathfrak{A}(\Lambda)$ and $D(\Lambda, d)$ intersects the disk $B(\bar{\varsigma}, 2 \rho)$, whereas $g_{d}=g$ admits analytic continuation to this disk. To finish the proof, it suffices to show that $D(\Lambda, d)$ does not contain the disk $B(\bar{\varsigma}, 2 \rho)$. Consider the points $z=r \bar{\varsigma}, r>1$. Since

$$
\left|\tilde{d}_{p, n}\right|=\left|\left(\begin{array}{c}
n(p) \\
n
\end{array}\right) \bar{\varsigma}^{n(p)-n}\right| \geq 1
$$

where $\left(\begin{array}{c}n(p) \\ n\end{array}\right)$ is the binomial coefficient, we obtain

$$
\begin{aligned}
\left|c_{p} \tilde{d}_{p, n} z^{n} \exp \left(\lambda_{k(p)} z\right)\right| & =r^{n}\left|\tilde{d}_{p, n}\right|\left|c_{p} z^{n} \exp \left(\lambda_{k(p)} z\right)\right| \geq\left|c_{p} z^{n} \exp \left(\lambda_{k(p)} z\right)\right| \\
& =\exp \left(-(1-1 / p)\left|\lambda_{k(p)}\right|\right) \exp \operatorname{Re}\left(\lambda_{k(p)} z\right) \\
& =\exp \left(-(1-1 / p)\left|\lambda_{k(p)}\right|\right) \exp \operatorname{Re}\left(\lambda_{k(p)} \bar{\varsigma}\right) \\
& =\exp \left(-(1-1 / p)\left|\lambda_{k(p)}\right|\right) \exp r\left|\lambda_{k(p)}\right| \operatorname{Re}\left(\bar{\varsigma} \lambda_{k(p)} /\left|\lambda_{k(p)}\right|\right) \\
& =\exp \left(-(1-1 / p)\left|\lambda_{k(p)}\right|\right) \exp r\left|\lambda_{k(p)}\right| \operatorname{Re}\left(\bar{\varsigma}\left(\varsigma+\xi_{p}\right)\right) \\
& =\exp \left(-(1-1 / p)\left|\lambda_{k(p)}\right|\right) \exp \left(\left|\lambda_{k(p)}\right|\left(r+\operatorname{Re}\left(\bar{\varsigma} \xi_{p}\right)\right)\right) \\
& \geq \exp \left(\left|\lambda_{k(p)}\right|\left((r-1)-\left|\xi_{p}\right|\right)\right) .
\end{aligned}
$$


Since $\left|\xi_{p}\right| \rightarrow 0$, this means that the series (5.2) diverges at all points $z=r \bar{\varsigma}, r>1$, i.e., the disk $B(\bar{\varsigma}, 2 \rho)$ is not included entirely in $D(\Lambda, d)$. This finishes the proof.

The next statement is a version of Theorem 3.1.

Lemma 5.2. Suppose that a sequence $\Lambda$ satisfies $m(\Lambda)=0$ and $N(\Lambda) \neq 0$. Then there exists a sequence $d \in \mathfrak{A}(\Lambda)$ of coefficients such that not all boundary points of the set $D(\Lambda, d)$ are singular for $g_{d}(z)$.

Proof. Passing to a subsequence, we may assume that $N(\Lambda)<\infty$ and still $N(\Lambda, \mathbb{C})=$ $N(\Lambda) \neq 0$. By the definition $N(\Lambda, \mathbb{C})$, it follows that for every $\delta \in(0,1)$ there is $\xi \in \mathbb{S}$ such that $N(\Lambda, \Gamma(\xi, \delta)) \neq 0$. We fix $\delta \in(0,1 / 8)$ and $\xi \in \mathbb{S}$ with $N(\Lambda, \Gamma(\xi, \delta / 6))$ strictly positive. Then $M_{\Lambda, \xi, \delta / 3}>0$ by Lemma 2.2. Referring to the definition of $M_{\Lambda, \xi, \delta / 3}$, we find an unbounded monotone increasing sequence $\left\{t_{p}\right\}$ of positive numbers such that

$$
\lim _{p \rightarrow \infty} \frac{M_{\Lambda}\left(t_{p} \xi, \delta / 3\right)}{t_{p}}>0 .
$$

It follows that, for all $p$ sufficiently large, the disk $B\left(t_{p} \xi, t_{p} \delta / 3\right)$ contains at least one point $\lambda_{k(p)}$ in the sequence $\left\{\lambda_{k}\right\}$. It can easily be seen that

$$
B\left(t_{p} \xi, t_{p} \delta / 3\right) \subset B\left(\lambda_{k(p)}, \delta\left|\lambda_{k(p)}\right|\right) .
$$

Therefore,

$$
\limsup _{p \rightarrow \infty} \frac{M_{\Lambda}\left(\lambda_{k(p)}, \delta\right)}{\left|\lambda_{k(p)}\right|} \geq \lim _{p \rightarrow \infty} \frac{M_{\Lambda}\left(t_{p} \xi, \delta / 3\right)}{(1-\delta / 3) t_{p}}>0 .
$$

Since $m(\Lambda)=0$ by assumption, we can remove (if necessary) some terms from the sequence $\left\{\lambda_{k(p)}\right\}$ to ensure the inequalities

$$
M_{\Lambda}\left(\lambda_{k(p)}, \delta\right)-m_{k(p)} \geq \widetilde{\beta}\left|\lambda_{k(p)}\right|, \quad p=1,2, \ldots
$$

for some $\widetilde{\beta}>0$. By Lemma 2.1, this implies the formula

$$
\ln \left|q_{\Lambda}^{k(p)}\left(\lambda_{k(p)}, \delta\right)\right| \leq \ln \frac{1}{3(1-\delta)}\left(M_{\Lambda}\left(\lambda_{k}, \delta\right)-m_{k}\right) \leq \ln \frac{8}{21} \widetilde{\beta}\left|\lambda_{k(p)}\right|=\beta\left|\lambda_{k(p)}\right| .
$$

As in Theorem 3.1, we may assume that each $\lambda_{k}$ belongs to a unique disk $B_{p}=$ $B\left(\lambda_{k(p)}, \delta\left|\lambda_{k(p)}\right|\right), p=1,2, \ldots$ Put

$$
g_{p}(z)=\frac{1}{2 \pi i} \int_{S\left(\lambda_{k(p)}, 4 \delta\left|\lambda_{k(p)}\right|\right)} \frac{\exp (\lambda z) d \lambda}{a_{p}\left(\lambda-\lambda_{k(p)}\right) q_{\Lambda}^{k(p)}(\lambda, \delta)}, \quad p=1,2, \ldots,
$$

where $a_{p} \geq 1$. As in Theorem 3.1, we obtain the inequalities

$$
\left|g_{p}(z)\right| \leq \exp \left(\operatorname{Re}\left(\lambda_{k(p)} z\right)+4 \delta\left|\lambda_{k(p)}\right||z|\right), \quad p=1,2, \ldots, \quad z \in \mathbb{C} .
$$

Consider the series

$$
g(z)=\sum_{p=1}^{\infty} c_{p} g_{p}(z)
$$

where $c_{p}=\exp \left(-2 \beta\left|\lambda_{k(p)}\right|\right), p=1,2, \ldots$ By (5.4), for all $z \in B(0, \alpha)$ with $\alpha=$ $2 \beta(1+4 \delta)^{-1}$, we have

$$
\begin{aligned}
\sum_{p=1}^{\infty}\left|c_{p} g_{p}(z)\right| & \leq \sum_{p=1}^{\infty} \exp \left(-2 \beta\left|\lambda_{k(p)}\right|+\operatorname{Re}\left(\lambda_{k(p)} z\right)+4 \delta\left|\lambda_{k(p)}\right||z|\right) \\
& \leq \sum_{p=1}^{\infty} \exp \left(-2 \beta\left|\lambda_{k(p)}\right|+(1+4 \delta)\left|\lambda_{k(p)}\right||z|\right)<\infty
\end{aligned}
$$


The last estimate holds true because $N(\Lambda)<\infty$ (i.e., $\left.\left|\lambda_{k(p)}\right| \geq c k(p), c>0\right)$. This means that the series (5.5) converges at every point of the disk $B(0, \alpha)$, and the convergence is uniform in every smaller disk. Therefore, $g(z)$ is analytic in $B(0, \alpha)$.

We define the coefficients $d=\left\{d_{k, n}\right\}_{k=1, n=0}^{\infty, m_{k}-1}$ precisely as we did in Theorem 3.1. Now, we choose $a_{p}, p=1,2, \ldots$. Specifically, $a_{p}$ is fixed in such a way that

$$
\max \left\{\left|\lambda_{k(p)}\right|^{-1}\left(\ln \left|b_{k, n}\right|-\ln a_{p}\right)\right\}=\beta,
$$

where the maximum is taken over all indices $k$ with $\lambda_{k} \in B_{p}$ and all $n=0,1, \ldots, m_{k}-1$. Then $a_{p} \geq 1$ by (5.3).

Now, we find the convergence domain for the series

$$
\sum_{k=1, n=0}^{\infty, m_{k}-1} d_{k, n} z^{n} \exp \left(\lambda_{k} z\right) .
$$

As in Theorem 3.1, we conclude that this series converges in the convex domain

$$
D(\Lambda, d)=\{z \in \mathbb{C}: \operatorname{Re}(z \lambda)<h(d, \lambda), \lambda \in \Theta(\Lambda)\}
$$

and diverges at each exterior point of this domain, except, possibly, the origin. Moreover, the function $h(d, \lambda)$ is defined by the formula

$$
h(d, \lambda)=\inf \liminf _{l \rightarrow \infty} \min _{0 \leq n \leq m_{s(l)}-1} \frac{\ln \left(1 /\left|d_{s(l), n}\right|\right)}{\left|\lambda_{s(l)}\right|}, \quad \lambda \in \Theta(\Lambda),
$$

where the infimum is taken over all subsequences $\left\{\lambda_{s(l)}\right\}$ of $\left\{\lambda_{k}\right\}$ for which $\lambda_{s(l)} /\left|\lambda_{s(l)}\right|$ tend to $\lambda$ as $l \rightarrow \infty$.

We estimate the quantity $h(d, \lambda), \lambda \in \Theta(\Lambda)$. Let $\lambda \in \Theta(\Lambda)$, and let a subsequence $\left\{\lambda_{s(l)}\right\}$ have the property that $\lambda_{s(l)} /\left|\lambda_{s(l)}\right|$ converges to $\lambda$ and $\lambda_{s(l)} \in B_{p(l)}, l=1,2, \ldots$ We have

$$
\begin{aligned}
\min _{0 \leq n \leq m_{s(l)}-1} \frac{\ln \left(1 /\left|d_{s(l), n}\right|\right)}{\left|\lambda_{s(l)}\right|} \geq \min _{\substack{k: \lambda_{k} \in B_{p(l)} \\
0 \leq n \leq m_{k}-1}} \frac{\ln \left(1 /\left|d_{k, n}\right|\right)}{\left|\lambda_{k}\right|} \\
=-\max _{\substack{k: \lambda_{k} \in B_{p(l)} \\
0 \leq n \leq m_{k}-1}} \frac{\left|\lambda_{k(p(l))}\right| \ln \left|d_{k, n}\right|}{\left|\lambda_{k(p(l))} \lambda_{k}\right|} \geq-\frac{1}{1 \pm \delta} \max _{\substack{k: \lambda_{k} \in B_{p(l)} \\
0 \leq n \leq m_{k}-1}} \frac{\ln \left|d_{k, n}\right|}{\left|\lambda_{k(p(l))}\right|} .
\end{aligned}
$$

Here the sign in the denominator is opposite to the sign of the maximum. By (5.6) and the definition of $c_{p}$, we obtain

$$
\begin{aligned}
\min _{0 \leq n \leq m_{s(l)}-1} \frac{\ln \left(1 /\left|d_{s(l), n}\right|\right)}{\left|\lambda_{s(l)}\right|} & \geq-\frac{1}{1 \pm \delta} \max _{\substack{k: \lambda_{k} \in B_{p(l)} \\
0 \leq n \leq m_{k}-1}} \frac{\ln \left|d_{k, n} / c_{p(l)}\right|+\ln \left|c_{p(l)}\right|}{\left|\lambda_{k(p(l))}\right|} \\
& =-\frac{1}{1 \pm \delta} \max _{\substack{k: \lambda_{k} \in B_{p(l)} \\
0 \leq n \leq m_{k}-1}}\left(\frac{\ln \left|d_{k, n} / c_{p(l)}\right|}{\left|\lambda_{k(p(l))}\right|}-2 \beta\right)=\frac{\beta}{1+\delta} \geq \frac{8 \beta}{9} .
\end{aligned}
$$

Thus, by the definition of $h(d, \lambda)$, we see that

$$
h(d, \lambda) \geq 4 \beta / 5, \quad \lambda \in \Theta(\Lambda) .
$$

Let $D=B(0,8 \beta / 9)$. Then $H_{D}(\lambda)=8 \beta / 9$ for all $\lambda \in \mathbb{S}$. The last-written inequality implies that $D(\Lambda, d)$ includes $D\left(\Theta(\Lambda)\right.$ ). As in Theorem 3.1, the function $g_{d}$ (the sum of the series (5.7)) coincides with $g$ on the intersection of the domains $D(\Lambda, d)$ and $B(0, \alpha)$, which is nonempty because $D(\Theta(\Lambda)) \cap B(0, \alpha) \neq \varnothing$.

As a result of our constructions, we have obtained a sequence $d=\left\{d_{k, n}\right\}$ of coefficients such that $d \in \mathfrak{A}(\Lambda)$ and $D(\Lambda, d)$ intersects the disk $B(0, \alpha)$ to which $g_{d}$ can be extended analytically. To finish the proof, it remains to show that $D(\Lambda, d)$ does not include $B(0, \alpha)$. 
We fix a subsequence $\left\{\lambda_{s(l)}\right\}, \lambda_{s(l)} \in B_{p(l)}, l=1,2, \ldots$, of $\left\{\lambda_{k}\right\}$ such that the numbers $\lambda_{s(l)} /\left|\lambda_{s(l)}\right|$ converge to a point $\xi \in \Theta(\Lambda)$ and, for $k=s(l)$ and some $n=n(l), l=1,2, \ldots$, the maximum in (5.6) is attained at $p=p(l)$, i.e.,

$$
\left|\lambda_{k(p(l))}\right| \ln \left|d_{s(l), n(l)} / c_{p(l)}\right|=\beta .
$$

By the definition of the numbers $c_{p}$, it follows that

$$
\begin{aligned}
\frac{\ln \left(1 /\left|d_{s(l), n(l)}\right|\right)}{\left|\lambda_{s(l)}\right|} & =\frac{-\left(\ln \left|d_{s(l), n(l)} / c_{p(l)}\right|+\ln \left|c_{p(l)}\right|\right)\left|\lambda_{k(p(l))}\right|}{\left|\lambda_{k(p(l))}\right|\left|\lambda_{s(l)}\right|} \\
& =\beta \frac{\left|\lambda_{k(p(l))}\right|}{\left|\lambda_{s(l)}\right|} \leq \frac{\beta}{1-\delta} \leq \frac{8 \beta}{7} .
\end{aligned}
$$

Consequently, $h(d, \xi) \leq 8 \beta / 7$. Let $z=r \bar{\xi}, 8 \beta / 7<r<4 \beta / 3$. Then $z \in B(0, \alpha)$ because $\alpha=2 \beta(1+4 \delta)^{-1} \geq 4 \beta / 3$. At the same time,

$$
\operatorname{Re}(z \xi)=\operatorname{Re}(r \bar{\xi} \xi)=r>8 \beta / 7>h(d, \xi),
$$

that is, $z \notin D(\Lambda, d)$. The lemma is proved.

Now we can state and prove the result announced at the beginning of the present section.

Theorem 5.1. The relations $N(\Lambda)=0$ and $S_{\Lambda}=0$ are necessary and sufficient for the existence domain of the sum of every series (1.1) with $D(\Lambda, d) \neq \varnothing$ to coincide with $D(\Lambda, d)$ (i.e., with the interior of the convergence domain for this series).

Proof. If $N(\Lambda)=0$ and $S_{\Lambda}=0$, then the existence domain of every series in question coincides with $D(\Lambda, d)$ by Corollary 4.4. Conversely, if the last feature occurs, then $m(\Lambda)=0$ by Lemma 5.1. But then $N(\Lambda)=0$ in view of Lemma 5.2 , and $S_{\Lambda}=0$ in view of Theorem 3.1

As has been shown above, $S_{\Lambda}=0$ provided $\lambda_{k}=k$ and $m_{k}=1$. So, Theorem 5.1 leads to the converse to Fabry's theorem.

Corollary 5.1. The existence domain of the sum of every series of the form (1.2) coincides with the disk of convergence of this series if and only if

$$
\lim _{n \rightarrow \infty} \frac{n}{k(n)}=0
$$

Remark. This result is a particular case of earlier results by Fuchs and Malliavin (see Chapter IX in the monograph [21]).

\section{$\S 6$. TOWARDS Ostrowski's THEOREM}

In this final section, we present the example mentioned in the Introduction, to indicate applicability limits for the result described in Ostrowski's theorem. This example is yet another version of Theorem 3.1. We start with constructing a suitable sequence $\left\{\lambda_{k}\right\}$. Fix two numbers $h, \alpha>0$ with $h \alpha<1$. We chose a monotone increasing sequence $\left\{\mu_{p}\right\}$ of positive numbers with $\mu_{p} / \mu_{p+1} \rightarrow 0$ as $p \rightarrow \infty$. Then we may assume that the intervals $\left[(1-h \alpha) \mu_{p}, \mu_{p}\right], p=1,2, \ldots$, are mutually disjoint. Let $s(p)$ be the integral part of $\alpha \mu_{p}$. Put $k(0)=0$ and $k(p)=k(p-1)+s(p)+1, p=1,2, \ldots$. Now, we define the sequence $\left\{\lambda_{k}\right\}$ in the following way:

$$
\lambda_{k(p)}=\mu_{p}, \quad \lambda_{k}=\lambda_{k(p)}-(k(p)-k) h, \quad k(p-1)<k<k(p), \quad p=1,2, \ldots
$$


Thus, the sequence $\Lambda=\left\{\lambda_{k}\right\}$ is split into groups $\Lambda_{p}=\left\{\lambda_{k(p-1)+1}, \ldots, \lambda_{k(p)}\right\}$, each group belonging to the corresponding interval $\left[(1-h \alpha) \mu_{p}, \mu_{p}\right], p=1,2, \ldots$. Since

$$
\lambda_{k(p-1)+1}-\lambda_{k(p)} \geq(1-h \alpha) \mu_{p+1}-\mu_{p}=\mu_{p+1}\left(1-h \alpha-\frac{\mu_{p}}{\mu_{p+1}}\right) \rightarrow \infty, p \rightarrow \infty,
$$

we see that $\liminf _{k \rightarrow \infty}\left(\lambda_{k+1}-\lambda_{k}\right)=h$. Furthermore,

$$
N(\Lambda)=\limsup _{k \rightarrow \infty} \frac{k}{\lambda_{k}}=\alpha \text {. }
$$

Indeed,

$$
\begin{aligned}
\lim _{p \rightarrow \infty} \frac{k(p)}{\lambda_{k(p)}} & =\lim _{p \rightarrow \infty} \frac{k(p-1)+s(p)+1}{\lambda_{k(p)}}=\lim _{p \rightarrow \infty} \frac{k(p-1)+\alpha \mu_{p}}{\lambda_{k(p)}} \\
& =\lim _{p \rightarrow \infty} \frac{k(p-1)+\alpha \lambda_{k(p)}}{\lambda_{k(p)}}=\lim _{p \rightarrow \infty} \frac{k(p-1)}{\lambda_{k(p)}}+\alpha .
\end{aligned}
$$

By construction, the quantity $k(p)$ coincides with the number of points $\lambda_{k}$ on the interval $\left[0, \mu_{p}\right]=\left[0, \lambda_{k(p)}\right]$. But this quantity does not exceed $\lambda_{k(p)} / h$. Therefore,

$$
0 \leq \lim _{p \rightarrow \infty} \frac{k(p-1)}{\lambda_{k(p)}} \leq \lim _{p \rightarrow \infty} \frac{\lambda_{k(p-1)} / h}{\lambda_{k(p)}}=0,
$$

whence

$$
\lim _{p \rightarrow \infty} \frac{k(p)}{\lambda_{k(p)}}=\alpha
$$

Let $k(p-1)<k<k(p)$; then

Consider the function

$$
\frac{k}{\lambda_{k}}=\frac{k(p)-s}{\lambda_{k(p)}-h s}
$$

$$
\varphi_{p}(x)=\frac{k(p)-x}{\lambda_{k(p)}-h x} .
$$

The sign of its derivative coincides with the sign of the number $h k(p)-\lambda_{k(p)}$, consequently, with the sign of $k(p) / \lambda_{k(p)}-1 / h$. Since $h \alpha<1$, the sign of the last quantity becomes negative for $p$ sufficiently large; see (6.1). Thus, for such $p$ we have

$$
\varphi_{p}(s)=\frac{k}{\lambda_{k}} \leq \varphi_{p}(0)=\frac{k(p)}{\lambda_{k(p)}} .
$$

Together with (6.1), this implies

$$
\limsup _{k \rightarrow \infty} \frac{k}{\lambda_{k}}=\limsup _{k \rightarrow \infty} \frac{k(p)}{\lambda_{k(p)}}=\alpha .
$$

Thus, $\Lambda$ satisfies the assumptions of the Ostrowski theorem. We fix a positive number $\delta<h \alpha$ and, for every $p=1,2, \ldots$, consider the function

$$
g_{p}(z)=\frac{1}{2 \pi i} \int_{S\left(\lambda_{k(p)}, 4 \delta \lambda_{k(p)}\right)} \frac{\exp (\lambda z) d \lambda}{a_{p}\left(\lambda-\lambda_{k(p)}\right) q_{\Lambda}^{k(p)}(\lambda, \delta)},
$$

where $a_{p} \geq 1$. In the same way as in Theorem 3.1 , we obtain the inequalities

$$
\left|g_{p}(z)\right| \leq \exp \left(\operatorname{Re}\left(\lambda_{k(p)} z\right)+4 \delta \lambda_{k(p)}|z|\right), \quad p=1,2, \ldots, \quad z \in \mathbb{C} .
$$

Let

$$
g(z)=\sum_{p=1}^{\infty} c_{p} g_{p}(z)
$$


where $c_{p}=\exp \left(-\beta \lambda_{k(p)}\right), p=1,2, \ldots$, and $\beta>0$ will be specified later. As in Lemma 5.2 ,

$$
\sum_{p=1}^{\infty}\left|c_{p} g_{p}(z)\right| \leq \sum_{p=1}^{\infty} \exp \left((\gamma-\beta) \lambda_{k(p)}+4 \delta \lambda_{k(p)}|z|\right)<\infty
$$

if $z$ belongs to the intersection of the disk $|z|<(\beta-\gamma) / 4 \delta$ and the half-plane $\operatorname{Re} z<\gamma$. Moreover, the series (6.2) converges uniformly on the compact subsets of this intersection, so its sum is analytic there. Furthermore, as in Lemma 5.2, the function $g(z)$ is analytic in the disk $B(0, \widetilde{\alpha}), \widetilde{\alpha}=\beta /(1+4 \delta)$. By construction, the quantity $M_{\Lambda}\left(\lambda_{k(p)}, \delta\right)-m_{k(p)}$ (to be denoted below by $s(p, \delta)$ ) coincides with the integral part of $\delta \lambda_{k(p)} / h$ if this number is not an integer, and with $\delta \lambda_{k(p)} / h-1$ otherwise. Then, by Lemma 2.1 , we obtain

$$
\ln \left|q_{\Lambda}^{k(p)}\left(\lambda_{k(p)}, \delta\right)\right| \leq s(p, \delta) \ln \frac{1}{3(1-\delta)} .
$$

It follows that

$$
\limsup _{p \rightarrow \infty} \frac{\ln \left|q_{\Lambda}^{k(p)}\left(\lambda_{k(p)}, \delta_{p}\right)\right|}{\lambda_{k(p)}} \leq \frac{\delta}{h} \ln \frac{1}{3(1-\delta)}=-\beta<0 .
$$

As in Theorem 3.1, we define numbers $a_{p} \geq 1$ and coefficients $d_{k}$ in such a way that

$$
\lim _{p \rightarrow \infty} \max _{k(p)-s(p, \delta) \leq k \leq k(p)}\left\{\left(\lambda_{k(p)}\right)^{-1} \ln \left|d_{k} / c_{p}\right|\right\}=\beta .
$$

Consider the Dirichlet series

$$
g(z)=\sum_{k=1}^{\infty} d_{k} \exp \left(\lambda_{k} z\right)
$$

As in Lemma 5.2, we use (6.3) to show that

$$
\begin{aligned}
\liminf _{k \rightarrow \infty} \frac{\ln \left(1 /\left|d_{k}\right|\right)}{\lambda_{k}} & =-\frac{1}{1 \pm \delta} \lim _{p \rightarrow \infty} \max _{k(p)-s(p, \delta) \leq k \leq k(p)} \frac{\ln \left|d_{k} / c_{p}\right|+\ln \left|c_{p(l)}\right|}{\lambda_{k(p)}} \\
& =-\frac{1}{1 \pm \delta} \lim _{p \rightarrow \infty(p)-s(p, \delta) \leq k \leq k(p)} \max _{k(p)}\left(\frac{\ln \left|d_{k} / c_{p}\right|}{\lambda_{k(p)}}-\beta\right)=0 .
\end{aligned}
$$

Consequently, the imaginary axis is the convergence line for the series (6.4).

It has been shown above that the sum $g(z)$ of the series $(6.4)$ is analytic in a neighborhood of the interval $[-i(\beta-\gamma) / 4 \delta, i(\beta-\gamma) / 4 \delta]$, where $\beta=(\delta / h) \ln (3(1-\delta))$. Thus, the distance between singular points of the sum of the series (6.4) on its convergence line attains the magnitude of order $O(1 / h)$; for $\alpha$ fixed and $h$ small, this is much larger than the radius $r(\alpha, h)$ in Ostrowski's theorem (which of order of $O(-\ln h)$. Furthermore, the function $g(z)$ is analytic also in the disk $B(0, \widetilde{\alpha})$, where $\widetilde{\alpha}=\beta /(1+4 \delta)$. Since $\delta$ has been chosen arbitrarily in the interval $(0, h \alpha)$, this means that Ostrowski's theorem concerns singular points of the series (6.4) that lie at a distance comparable with the upper density $\alpha$ of $\Lambda$ from the convergence line.

\section{REFERENCES}

[1] A. V. Bratishchev, Keith bases, entire functions and their applications, Dissertation, Rostov on Don, 1995. (Russian)

[2] O. A. Krivosheyeva, Series of exponential monomials in complex domains, Vestnik Ufim. Aviats. Tekhn. Univ. 9 (2007), no. 3 (21), 96-103. (Russian)

[3] J. Hadamard, Essai sur l'étude des fonctions données par leur développement de Taylor, J. Math. Pures Appl. (4) 8 (1892), 101-186.

[4] E. Fabry, Sur les points singuliers d'une fonction donnée par son développement en série et l'impossibilité du prolongement analytique dans des cas très généraux, Ann. Sci. Ecole Norm. Sup.

(3) 13 (1896), 367-399. MR:1508933 
[5] G. Polya, Über die Existenz unendlich vieler singulärer Punkte auf der Konvergenzgeraden gewisser Dirichletscher Reihen, S.-B. Preuss. Akad., Phys.-Math. Kl. (1923), 45-50.

[6] _ Eine Verallgemeinerung des Fabryschen Lückensatzes, Nachr. Ges. Wiss. Göttingen, Math.-Phys. Kl. 2 (1927), 187-195.

[7] A. F. Leont'ev, Exponential series, Nauka, Moscow, 1976. (Russian) MR0584943 (58:28451)

[8] V. Bernstein, Leçons sur les progrès récents de la théorie des séries de Dirichlet, Gauthier-Villars, Paris, 1933.

[9] A. F. Leont'ev, Entire functions. Series of exponentials, Nauka, Moscow, 1983. (Russian) MR0753827 (86j:30005)

[10] A. Ostrowski, Uber die analytische Fortsetzung von Taylorschen und Dirichletschen Reihen, Math. Ann. 129 (1955), 1-43. MR0069878 (16:1094a)

[11] G. L. Lunts, On Dirichlet series with complex exponents, Mat. Sb. (N. S.) 67 (1965), no. 1, 89-134. (Russian) MR0196047 (33:4241)

[12] _ Dirichlet series with a non-measurable sequence of complex exponents, Mat. Sb. (N. S.) 68 (1965), no. 1, 58-62. (Russian) MR0204626 (34:4465)

[13] A. S. Krivosheyev, A criterion for the fundamental principle for invariant subspaces, Dokl. Akad. Nauk 389 (2003), no. 4, 457-460. (Russian) MR2042069

[14] _. The fundamental principle for invariant subspaces in convex domains, Izv. Ross. Akad. Nauk Ser. Mat. 68 (2004), no. 2, 71-136; English transl., Izv. Math. 68 (2004), no. 2, 291-353. MR2058001 (2006g:30061)

[15] B. Ya. Levin, Distribution of zeros of entire functions, Gostekhizdat, Moscow, 1956; English transl., Transl. Math. Monogr., vol. 5, Amer. Math. Soc., Providence, RI, 1980. MR0087740 (19:402c) MR $0589888(81 \mathrm{k}: 30011)$

[16] K. Leichtweiss, Konvexe Mengen, Springer-Verlag, Berlin-New York, 1980. MR0586235 (81j:52001)

[17] P. Lelong and L. Gruman, Entire functions of several complex variables, Grundlehren Math. Wiss., Bd. 282, Springer-Verlag, Berlin, 1986. MR0837659 (87j:32001)

[18] V. V. Napalkov, Convolution equations in multidimensional spaces, Nauka, Moscow, 1982. (Russian) MR0678923 (86g:46054)

[19] I. F. Krasichkov-Ternovskiu,, Invariant subspaces of analytic functions. III. Extension of spectral synthesis, Mat. Sb. (N. S.) 88 (1972), no. 3, 331-352; English transl., Math. USSR-Sb. 17 (1972), no. 3, 327-348. MR0422637 (54:10623)

[20] S. Mandelbrojt, Séries adhérentes, régularisation des suites, applications, Gauthier-Villars, Paris, 1952. MR0051893(14:542f)

[21] P. Koosis, The logarithmic integral. II, Cambridge Stud. in Adv. Math., vol. 21, Cambridge Univ. Press, Cambridge, 1992. MR 1195788 (94i:30027)

Bashkir State University, Zaki Validi st. 32, Ufa 450074, Russia

E-mail address: kriolesya2006@yandex.ru

Received 17/JUL/2009

Translated by S. KISLYAKOV 